\title{
Synthesis of Regulation Compliant Business Processes*
}

\author{
Nina Ghanbari Ghooshchi ${ }^{1,2}$, Nick van Beest ${ }^{1}$, Guido Governatori ${ }^{1}$, Francesco Olivieri ${ }^{1}$ \\ ${ }^{1}$ Data61, CSIRO, Dutton Park, Australia \\ \{nick.vanbeest, guido.governatori, francesco.olivieri\}@data61.csiro.au \\ ${ }^{2}$ Griffith University, Australia \\ nina.ghanbari@griffithuni.edu.au
}

\begin{abstract}
Organisations have to cope with large numbers of business rules and existing regulations governing the business in which they operate. Such rules are difficult to maintain due to their size and complexity, and it is increasingly challenging to ensure that each business process adheres to those rules. As such, automated extraction of business processes from rules has three clear advantages: (1) visualisation of all possible executions allowed by the rules, (2) automated execution and compliance by design, (3) identification of "inefficiencies" in the business rules. Existing approaches, however, only allow for the generation of partial traces based on input specifications and cannot handle many different input cases resulting in a full process. This paper presents a formal method to visualise and operationalise such sets of rules as a verifiable business process that is compliant by design, which allows us to analyse all possible execution paths. Additionally, we formally prove correctness of the business processes generated by our method. The approach is implemented in a tool and evaluated on both performance and correctness, showing that even for highly complex sets of rules the approach performs well and outperforms a well-known state-of-the-art approach. Evaluation on a real-life process shows the feasibility of the presented approach.
\end{abstract}

\section{INTRODUCTION}

It is a common scenario that organisations develop business rules manuals for their operations. Such business rules may specify constraints that apply to their business processes (e.g. a customer has to be older than 18 in order to be eligible for a loan). As organisations grow, so do their processes and business rules. As a consequence, the number of business rules is generally large [2].

In addition, organisations have to comply with regulations governing the business in which they operate. These regulations (as the etymology of the name itself, regula in latin means rule) consist of rules as well, and the compliance rules affect the procedures and processes described by the business rules. Although the combination of business rules and compliance rules allows organisations to determine whether such procedures and processes are compliant with the regulatory requirements, in general, for large rule sets the relationships among the rules (and the activities and conditions they describe) are difficult to understand and maintenance of those rule sets is complicated [2]. Furthermore, it is difficult to ensure that each business process adheres to those rules [3].

\footnotetext{
${ }^{*}$ This paper extends the work presented in [1] in the following ways: (i) it provides an updated and more efficient algorithm, (ii) it provides formal proofs of correctness of the algorithms, and (iii) it includes an extensive evaluation of the approach on both synthetic and real-life processes.
}

However, the combination of these rules implicitly contains the processes for organisations, as the constraints are specified by those rules. The extraction of business processes from rules has a number of clear advantages: (1) it helps to visualise all possible executions allowed by the rules, (2) the process can be implemented by a workflow engine (e.g. [4]) for automated execution and compliance by design, (3) it helps to identify "inefficiencies" in the business rules (e.g. rules with side effects that do not contribute to the goals of a process are visualised as separate paths in the process that only merge at the end, multiple rules for the same goal are visualised as redundant transitions, etc).

As a starting point to create our business processes we adopt a rule-based formalism, because rules are well suited to describe both the external constraints regulating the setting, and which actions the agent may undertake. In this way, rules are able to define relationships among the tasks to be executed to achieve a goal, are useful in understanding the behaviour of such tasks. By shifting the focus from the entire process to its basic building blocks, it can be identified which preconditions trigger the activation of a task, as well as what happens once a task completes its execution. This way of describing processes provides knowledge concerning the reasons why to execute a task instead of another and, implicitly, defines the control flow of a process as all the situations that do not violate any of the given relationships.

Existing approaches to transform rules into readable business process models, however, only produce partial traces based on input specifications [5][6], cannot handle many different input cases resulting in a full process [7] or duplicate tasks to represent every possible trace [8]. Therefore, to tackle these problems, this paper presents a new formal method to visualise and operationalise such sets of rules as a verifiable business process (i.e., it is possible to check whether the process is sound [9] and compliant [10]), by generating individual Petri nets for each each case and subsequently merging them in such a way that duplication of tasks is avoided. The generated business process is compliant by design and allows us to analyse all possible execution paths within the rules (i.e. supporting all input cases). Furthermore, the generated business process is tailored to support and adhere to the business rules and regulations, as it is specifically designed to exactly represent the behaviour allowed by the rules.

The remainder of this paper is structured as follows. In Section II, we discuss the background with respect to rules for declarative processes. Subsequently, Section III describes 
the preliminary definitions related to Petri nets. The method and the corresponding algorithms are described in Section IV, along with a running example, followed by a formal proof of correctness of the generated Petri net. The presented approach is evaluated in Section V. Lastly, Section VI presents the related work, while Section VII concludes the paper and discusses future work.

\section{Rules for Declarative Processes}

\section{A. Background}

Governatori et al. [11][12] proposed an agent-oriented rule language for the declarative specifications of norm and goal compliant business processes. The main idea is that the set of rules can be partitioned into three subsets: a set of rules corresponding to the norms governing a process, a set of rules encoding the objectives/goals of an organisation to fulfil in their processes and, finally, a set of rules describing the "capabilities" of an organisation. The intuition behind the capability rules being that they model the set of activities/tasks an organisation is able to carry out, the preconditions required for each task, the effects of such tasks, and the relationships among tasks. The language upon which the rules are defined consists of a set of two types of literals: condition literals and task literals. The condition literals encode the preconditions and effects of tasks or, in general, state variables for a process, while each task literal corresponds to a task that could occur in a process. The three sets of rules have the form of If ...then statements that can containing expressions like: if $c_{1}, t_{1}$ then OBL $t_{2}$ meaning that that there is a norm that specify that the execution of task $t_{1}$ in presence of condition $c_{1}$ makes it obligatory to execute task $t_{2}$; and if $\mathrm{OBL} c, t_{i}$ then $t_{j}$ whose reading is: task $t_{j}$ requires task $t_{i}$ to be executed when $c$ is obligatory.

The logic proposed by [11][12] was devised to determine if, given a set of facts, it was possible to execute a process that achieve the objective of an organisation and at the same time complying with the norms governing the process. The underlying idea is that, given a set of facts, a derivation can be generated where all applicable rules fire and their conclusions have been added to the derivation. This derivation contains all tasks that are executable given the set of facts, which can be considered as the input for a process case (and instance of a business process). In addition, the derivation contains the literals corresponding to the conditions to trigger the execution of tasks or for activating obligations, the effects of the tasks, the obligations in force, and the expected goals. Note that while obligations and goals determine whether an execution of the process is compliant and meet the organisation objectives (thus influencing the activities or tasks included in the process), they are neither actions nor tasks. Therefore, rules for goals and norms do not directly contribute to the structure of the process: they only provide constraints on the tasks that can appear in a derivation. Goals and obligations can thus be considered as special kinds of conditions. Accordingly, if one ignores obligations, goals and the condition literals from a derivation, then a derivation is a sequence of tasks satisfying the constraints defined by the rules. This means that it is equivalent to a plan as defined in classical planning [13]. For these reasons, in this paper, we concentrate only on the capability rules. Note, that, while the approach was originally intended for the logic proposed in [11][12], the method we are going to present in this paper can be used with any rule language satisfying derivation conditions described in the remainder of this section.

\section{B. Technical definitions}

Formally a (capability) rule is an expression $r: l_{1}, \ldots, l_{n} \Rightarrow$ $l_{n+1}$, where $r$ is a label that uniquely identifies the rule, and each literal $l_{i}$ is drawn from the set of literals Lit $=$ Prop $\cup\{\neg p \mid p \in$ Prop $\}$; Prop is a set of propositional atoms representing conditions $c_{i}$ and tasks $t_{j}$. This form has the clear advantage that it immediately relates preconditions to the corresponding effect of performing the particular action. More specifically, we can identify the following three patterns. (1) $t \Rightarrow c$, where we can look at $c$ as an effect of performing task $t$; thus the effect represented by $c$ holds after the execution of task $t$. (2) The pattern $c_{1}, \ldots c_{n} \Rightarrow t$ indicates that $c_{1}, \ldots c_{n}$ are preconditions for tasks $t$, and task $t$ will be executed after the preconditions hold. (3) $t_{1}, \ldots, t_{n} \Rightarrow t$ specifies that the combination of tasks $t_{1}, \ldots, t_{n}$ triggers task $t$, and that task $t$ appears in the process, if $t_{1}, \ldots, t_{n}$ appear in the process (before $t$ ). In other words, this pattern describes relationships and dependencies among tasks in a process. In the rule given above, the meaning is that execution of tasks $t_{1}, \ldots, t_{n}$ is required to trigger the execution of task $t$. It is possible to have negations of task literals, e.g. $\neg t$, meaning $t$ does not appear in the process. Accordingly, a rule $t_{1}, c \Rightarrow \neg t_{2}$ means if task $t_{1}$ is executed, and $c$ holds, then task $t_{2}$ does not appear in the process; similarly, $\neg t_{1} \Rightarrow t_{2}$ means that if task $t_{1}$ does not appear in the process, then task $t_{2}$ is executed.

The rules are then used to form (logical) derivations, where a derivation $D$, given a set of facts $F$ represented as literals, is a sequence of literals $D(1), \ldots, D(n)$, such that if $D(m+1)=l$ then either $l \in F$ or there is a rule $r: l_{1}, \ldots, l_{k} \Rightarrow l$ such that for all $i \leq k, l_{i} \in D[1 . . m]$, where $D[1 . . m]$ is the initial sequence of length $m$ of $D$.

The rules presented above can be linked to the sequential, parallel, and alternative patterns typical of business process modelling techniques to those that can be found in a logical derivation. Indeed, assume tasks $A$ and $B$ concur to obtain the resources needed for task $C$ to start its execution. This means that $C$ may bring about its effects only when both $A$ and $B$ have finished, and that $A$ and $B$ have no precedence order with respect to one another, that is they can be executed in parallel. From a logical perspective, all this information can simply be represented by a rule whose premises are literals $A$ and $B$ and which conclusion is $C$, i.e., something of the form ' $A, B \Rightarrow C$ '. As such, a derivation can encode a possible order in which the tasks are executed to achieve a particular business goals according to the constraints specified by the rules themselves.

Note that, while the set of tasks triggered by a case (set of facts) is unique, multiple derivations are possible. For example, given the rule $t_{1}, t_{2} \Rightarrow t_{3}$, the order in which $t_{1}$ and $t_{2}$ are executed does not matter. Accordingly, both $t_{1}, t_{2}, t_{3}$ and $t_{2}, t_{1}, t_{3}$ are valid derivations (and, consequently, plans conforming to the specification given by the rule). This means that, given a case, we can generate a set of plans corresponding to it, which can be understood as alternative ways in which the process 
can be executed. Using the idea that a business process can be understood by a set of traces (where a trace is a sequence of tasks), we can establish a connection between a set of plans and a business process, where a process provides a concise (formal and graphical) representation of a set of plans, which are obtained from a single case and are combined using constructions modelling and-splits and and-joins. Moreover, given a set of rules, it is possible to give as input mutually exclusive sets of facts, where, for instance, each set of facts corresponds to a common set of instances for the process. For each case, a corresponding set of plans is created, where the mutually exclusive cases are subsequently merged, adopting xor-splits and xor-joins.

Let us explain how the derivation mechanism works by considering the following example. Consider the set of rules $R=\left\{r_{0}: A \Rightarrow C, r_{1}: B \Rightarrow D, r_{2}: C, D \Rightarrow E\right\}$. and the facts (or case) $A, B$. It is easy to see that all the rules are applicable and actually fire producing the following six derivations:
(1) $A, B, C, D, E$
(4) $B, A, C, D, E$
(2) $A, B, D, C, E$
(5) $B, A, D, C, E$
(3) $A, C, B, D, E$
(6) $B, D, A, C, E$

Some considerations: $A$ and $B$ have no precedence order between each other. The same happens for the tuples: $(C, D)$, $(A, D)$, and $(B, C)$. However, $A$ always precedes $C, B$ always precedes $D$, and so do $C$ and $D$ for $E$. It is straightforward to see that such derivations can be visualised graphically in the Petri net below:

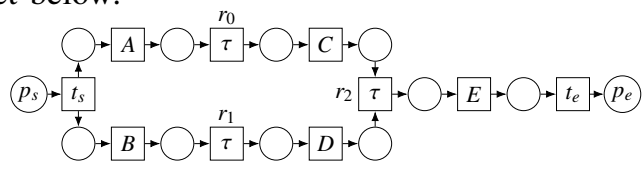

It is easy to see that for larger processes the amount of derivations is combinatorial and, therefore, intractable. In this paper, we present a method that directly uses the rules to derive the process, omitting the need for generating all derivations.

\section{Petri nets}

Business processes can be modelled by using many different notations. Often these notations require further formalisation before formal verification can be applied. Petri nets (PN) are a popular modelling language used to formalise business processes [14]. Petri nets are mathematical models for the description of distributed systems. A Petri net [15] is a tuple $(P, T, A, \lambda)$, where $P$ is a set of places and $T$ is a set of transitions, such that $P \cap T=\emptyset$. Arcs form directed edges between place-transition pairs, such that $A \subseteq(P \times T) \cup(T \times P)$. $\lambda: P \cup T \rightarrow \mathcal{L}$ is a labelling function. The Petri net state, often referred to as the net marking, $M: P \rightarrow \mathbb{N}_{0}$ is a function that associates a place $p \in P$ with a natural number (viz., place tokens). A marked net $N=\left(P, T, A, \lambda, M_{0}\right)$ is a Petri net $(P, T, A, \lambda)$ together with an initial marking $M_{0}$.

Places and transitions are referred to as nodes. The preset of a node is denoted by $\bullet y=\{x \in P \cup T \mid(x, y) \in A\}$, and the postset of a node is denoted by $y \bullet=\{z \in P \cup T \mid(y, z) \in A\}$. Two places $p_{i}$ and $p_{j}$ are equal (i.e. $p_{i}=p_{j}$ ) iff $\bullet p_{i}=\bullet p_{j} \wedge$ $p_{i} \bullet=p_{j} \bullet$. Two transitions $t_{i}$ and $t_{j}$ are equal (i.e. $t_{i}=t_{j}$ ) iff $\lambda\left(t_{i}\right)=\lambda\left(t_{j}\right)$.

If $\forall p \in \bullet t: M(p)>0, t$ is said to be enabled. The firing of $t$, denoted by $M \stackrel{t}{\longrightarrow} M^{\prime}$, leads to a new marking $M^{\prime}$, with
$M^{\prime}(p)=M(p)-1$ if $p \in \bullet t \backslash \bullet, M^{\prime}(p)=M(p)+1$ if $p \in t \bullet \backslash \bullet t$, and $M^{\prime}(p)=M(p)$ otherwise. The marking $M_{n}$ is said to be reachable from $M$ if there exists a sequence of transition firings $\sigma=t_{1} t_{2} \ldots t_{n}$ such that $M \stackrel{t_{1}}{\longrightarrow} M_{1} \stackrel{t_{2}}{\longrightarrow} \ldots \stackrel{t_{n}}{\longrightarrow} M_{n}$.

A marking $M$ of a net is $n$-bounded if $\forall p: M(p) \leq n$. A Petri net $N$ is $n$-bounded if all its reachable markings are $n$ bounded. A Petri net $N$ with initial marking $M_{0}$ is live if it is possible to eventually fire any transition of $N$ by progressing through some further firing sequence [16]. Five categories of liveness are defined in [16]. For the needs of this paper, we can restrict ourselves to $L_{1}$-live: $t$ can be fired at least once in some firing sequence following $M_{0}$. A Petri net $N$ with initial marking $M_{0}$ is deadlock-free iff $\forall M: M_{0} \rightarrow \ldots \rightarrow M$, $\exists M^{\prime}: M \rightarrow M^{\prime}$. That is, any reachable marking $M$ enables some transition $t$, such that there exists a subsequent marking $M^{\prime}$, where $M^{\prime} \neq M$. A Petri net $N$ with initial marking $M_{0}$ is sound iff it is bounded, live and deadlock-free.

A trace is a sequence $\lambda\left(t_{1}\right), \lambda\left(t_{2}\right), \ldots$ such that $\sigma=t_{1}, t_{2}, \ldots$ is a sequence of firing transi-

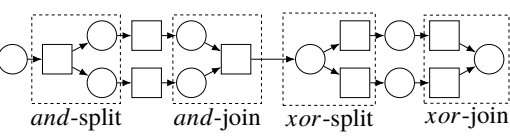
tions. However, certain control-flow behaviour (like certain exclusive (xor) and concurrent (and) constructs) requires additional transitions that do not correspond to a task literal. These transitions are commonly referred to as silent or $\tau$ transitions [17]. To improve clarity and for ease of reference, we will use a label for each $\tau$ transition as well throughout the paper. As such, the set of transition labels $\mathcal{L}$ comprises both labels corresponding to task literals and labels corresponding to $\tau$ transitions. A visible trace is a trace where all $\tau$ transitions have been removed (maintaining the order of the transitions representing task literals). For the remainder of this work, we refer to visible traces as traces.

\section{From Rules to Petri Nets}

\section{A. Method}

In order to transform a set of rules $R$ representing the regulations governing an organisation to a Petri net $N$, a number of steps are required, as shown graphically in Figure 1.

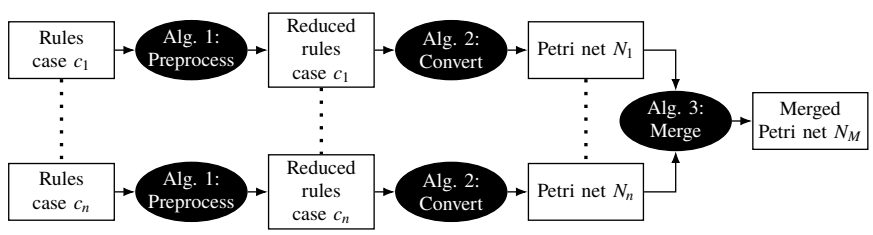

Fig. 1: Method overview.

First, some preprocessing of the rules is required to take into account only those rules that define the possible executions in the process. That is, we restrict the analysis to what we called capability rules. In case the language contains rules for obligations, goals and we transform obligations and goals in capability rules into conditions. From the resulting set of (capability) rules, we remove all rules with literals that are not in the set of all conclusions, as these have no effect on the resulting process.

The resulting set of (preprocessed) rules will be used to produce the Petri net. However, only tasks that are potentially executed should be represented by a transition in $N$. As negative tasks represent the absence of a task in the execution, 
these can be eliminated from the antecedents of the rules (e.g. $A, \neg B \Rightarrow C$ would be $A \Rightarrow C$ ). These negative tasks can be treated as additional "conditions", which are satisfied automatically as they do not appear in the resulting net $N$ and can, therefore, not be executed. Positive tasks that are in the set of facts represent the initial state. Positive tasks from the initial state that are in the antecedent of a rule can be removed as well, as they hold by default. In addition, rules containing a negative task in the consequence (e.g. $A \Rightarrow \neg B$ ) can be removed, as these rules result in the absence of a task, which is automatically satisfied by not including the respective transition in the resulting net $N$. The removal of negative tasks is shown in Algorithm 1 below.

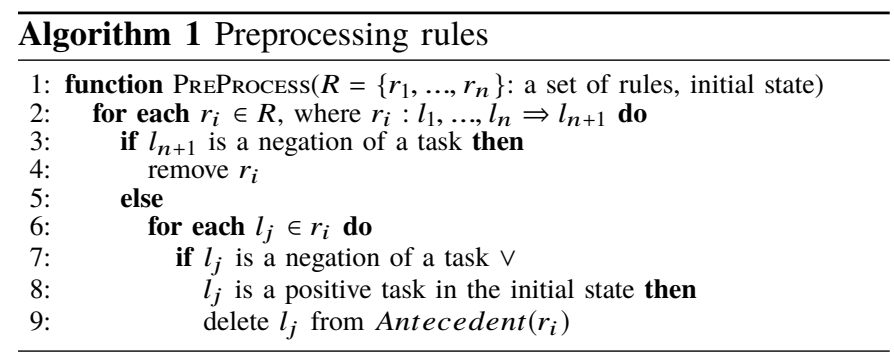

\section{B. Obtaining a Petri net from rules}

Once we have obtained the reduced rule set for each case $c s_{i}$, we can subsequently transform each set of reduced rules to a Petri net $N_{i}$ corresponding to that case by using Algorithm 2 . That is, the traces of each $N_{i}$ contain exactly all possible derivations from the rules of its corresponding case $c s_{i}$.

$$
p_{s}=t_{s}
$$

The net $N_{i}$ starts with an initial place $p_{s}$ and subsequent transition $t_{s}$ (Line 6-8). From there, the initial rules (i.e. rules with empty antecedent) are added (Line 9-11). For example, a rule $r_{0}: \Rightarrow A$ would add an additional place and a transition $A$ after $t_{s}$.

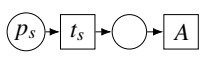

A rule $r_{1}: A \Rightarrow B$ first adds a $\tau$ transition representing $r_{1}$, followed by a place and a transition $B$ after $A$, to realise the sequence from $A$ to $B$ induced by $r_{1}$. Similarly, adding a rule $r_{2}: A \Rightarrow C$ would add another outgoing place from $\mathrm{A}$ followed by a similar construct as created by $r_{1}$ leading to a transition $C$.

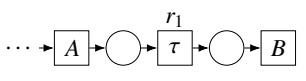

A rule with multiple tasks in its antecedent, e.g. $r_{3}: B, C \Rightarrow$ $D$, results in an $a n d$-join of $B$ and $C$ to $D$ (Line 13-17).

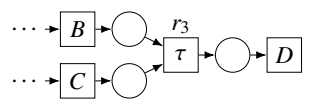

Finally, all transitions $t_{i}$ with $t_{i} \bullet=\emptyset$ are joined into a common end transition $t_{e}$ (Line 31-32), which leads to the sink place $p_{e}$ (Line 6-8).

$$
\cdots \rightarrow \bigcirc-t_{e} \rightarrow P_{e}
$$

When merged together, the resulting net can be visualised as follows:

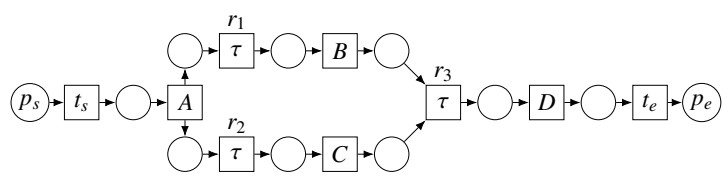

These basic principles work well for simple scenarios that result in a structured Petri net (i.e. every split has its matching join). However, when two concurrent paths lead into the same transition without synchronisation, this may cause the transition to be executed more than once.

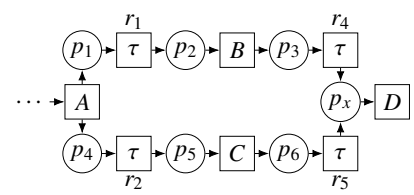

This can easily be illustrated by replacing $r_{3}$ in the previous example with $r_{4}: B \Rightarrow D$ and $r_{5}: C \Rightarrow D$. It is straightforward to see that the execution of $A$ activates both branches, resulting in two tokens in $p_{x}$. Therefore, an additional construct is required to allow either $r_{4}$ or $r_{5}$ to produce a token for $p_{x}$ and remove the remaining tokens.

To allow either $r_{4}$ or $r_{5}$ to produce a token for $p_{x}$, auxiliary transitions $r_{4}^{\prime}$ and $r_{5}^{\prime}$ are added in Line 23-25. A common input place $p_{\oplus}$ is introduced for $r_{4}^{\prime}$ and $r_{5}^{\prime}$, to allow only one of them to fire (Line 19, 21 and 26). Auxiliary transitions $r_{i}^{\prime \prime}$ are added to clean up remaining tokens (Line 23). The inputs of auxiliary transition $r_{i}^{\prime \prime}$ are from all $r_{j}^{\prime}$ s except $r_{i}^{\prime}$ (Line 2728). When $r_{i}^{\prime}$ fires, $r_{i}^{\prime \prime}$ cleans up remaining tokens produced by all $r_{j}^{\prime}$ s. As a result, firing of $r_{4}^{\prime}$ coincides with $r_{4}^{\prime \prime}$ and firing of $r_{5}^{\prime}$ coincides with $r_{5}^{\prime \prime}$.

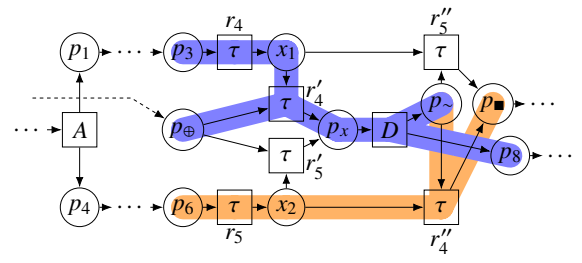

Let us assume a scenario where both $r_{4}$ and $r_{5}$ have fired, resulting in a token in $x_{1}$ and a token in $x_{2}$ respectively. If $r_{4}^{\prime}$ fires, it consumes tokens from $p_{\oplus}$ and $x_{1}$ and produces a token to $p_{x}$ to allow $D$ to fire and produce a token to $p_{8}$ and auxiliary place $p_{\sim}$ (highlighted in purple). The remaining token in $x_{2}$ is consumed along with the token in $p_{\sim}$ by $r_{4}^{\prime \prime}$, which produces a token for $p_{\square}$ (highlighted in orange). Place $p_{\sim}$ is the common place for auxiliary transitions $r_{i}^{\prime \prime}$ allowing only one of them to execute (Line 29-30). Place $p_{8}$ is then connected to any subsequent rules having $D$ in their antecedent (e.g. $r_{6}: D \Rightarrow$ $E$ ), whereas $p_{\boldsymbol{m}}$ is connected to the final transition to ensure that all remaining tokens are consumed (Line 33-34).

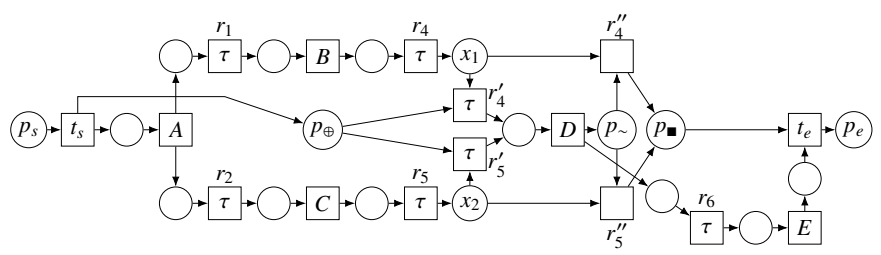

Fig. 2: Petri net showing the merge of two branches without synchronisation.

This construct ensures that, while having two rules producing $D$, only one execution of $D$ is possible, without having 
any orphaned tokens after finishing execution of the process. The entire net can be visualised as shown in Figure 2.

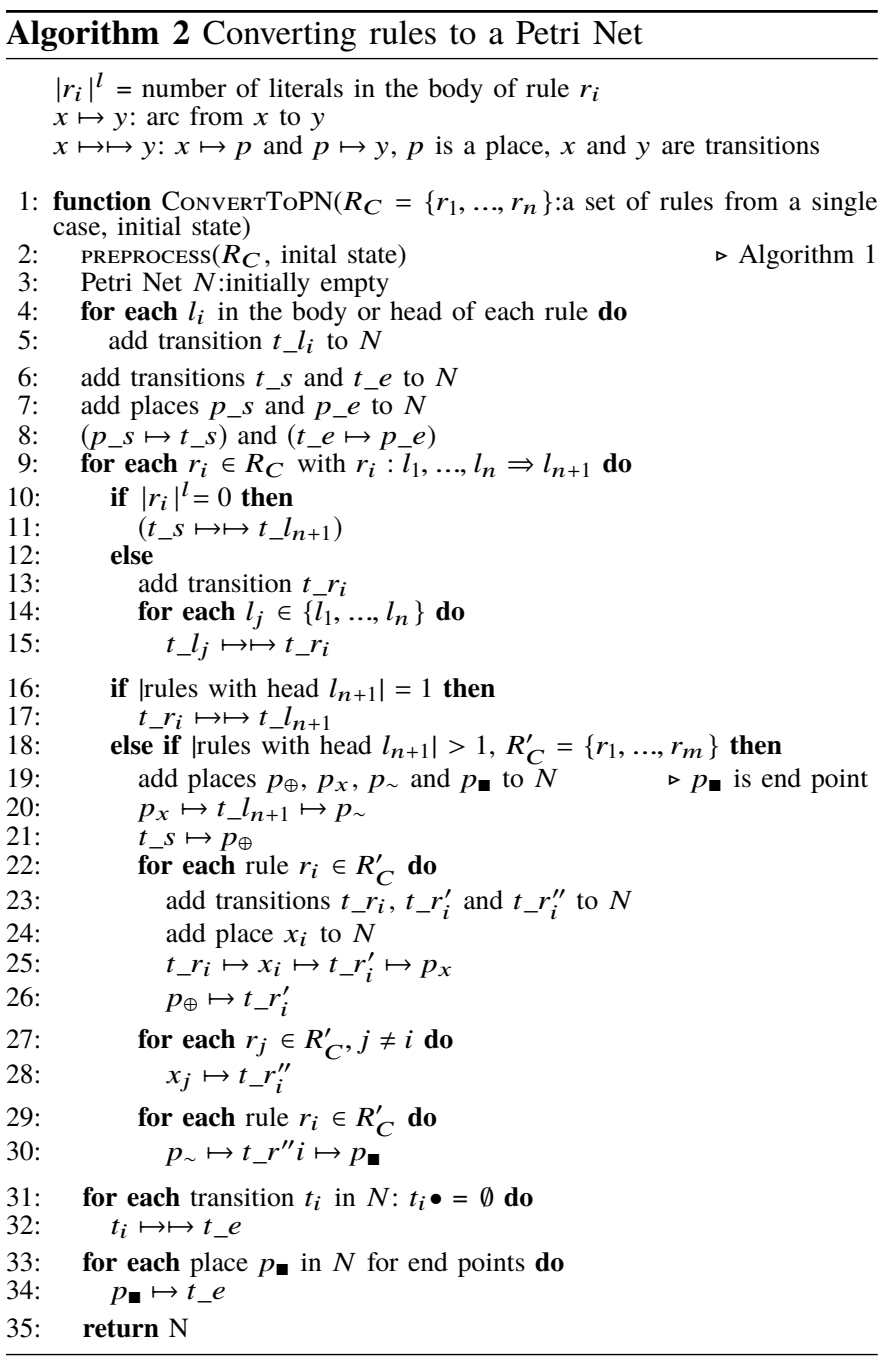

\section{Merging cases}

The algorithm described in the previous subsection is used for transforming individual cases into Petri nets. The final step is to merge the Petri nets of these individual cases into a combined Petri net that represents the entire process without duplicating transitions, which is achieved through Algorithm 3.

Let us illustrate the merging with a small example, where we have two different cases: Case $c s_{1}=\left\{r_{1}: \Rightarrow A, r_{2}: A \Rightarrow C, r_{3}\right.$ : $C \Rightarrow D\}$ and Case $c s_{2}=\left\{r_{4}: \Rightarrow B, r_{5}: B \Rightarrow C, r_{6}: C \Rightarrow E\right\}$. The Petri nets $N_{1}$ and $N_{2}$, representing $c s_{1}$ and $c s_{2}$ respectively, are shown graphically in Figure 3. Note that the two cases differ on the first and final transition to be executed, but contain the mutual transition $C$ in between.

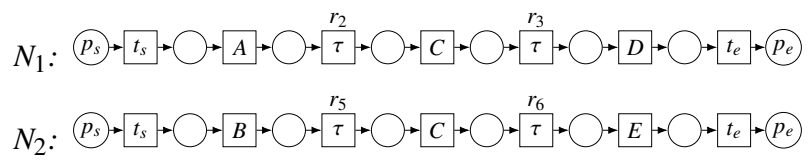

Fig. 3: Petri nets $N_{1}$ and $N_{2}$ for cases $c s_{1}$ and $c s_{2}$.

Furthermore, $A$ co-occurs with $D$ and $B$ co-occurs with $E$, but $A$ and $E$ or $B$ and $D$ cannot occur in the same trace. As such, $p_{s}$ and $t_{s}$ are common, where the outgoing place (labeled $x_{o r}$ ) of $t_{s}$ marks the split to either $A$ (case $c s_{1}$ ) or $B$ (case $\left.c s_{2}\right)$. Next, $A$ leads to $C$ via $r_{2}$, whereas $B$ leads to $C$ via $r_{5}$, having $\mathrm{xor}_{2}$ as $x$ or-join place.

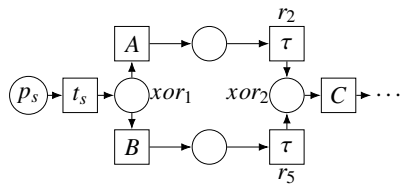

Similarly, from $C$ there exist two rules $\left(r_{3}\right.$ and $r_{6}$ ), leading to $D$ and $E$ respectively. Together with the common end transition $t_{e}$ and place $p_{e}$, the resulting Petri net would look as follows:

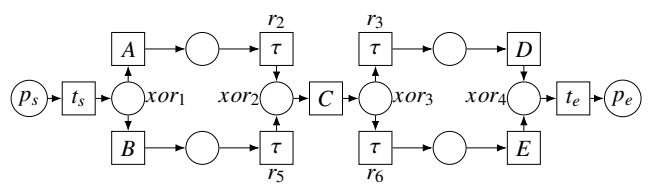

Fig. 4: Merged Petri net $N_{M}$ without preserving dependencies.

However, we want to obtain a condition-free representation of subsequent exclusive branches that maintain their dependencies without duplicating transitions. Although transition $C$ should not be duplicated in the merged Petri net, dependencies between $A$ and $D$ should remain intact (similar for $B$ and $E$ ). In the example shown in Figure $4, A$ could be followed by $E$, which is incorrect as both correspond to different cases. ${ }^{1}$

When the presets and/or postsets of common transitions (i.e. $t_{s}, C$ and $t_{e}$ ) are different, additional $\tau$ transitions are required to enforce these case-specific dependencies. For instance, transition $t_{s}$ has different postsets for $c s_{1}$ and $c s_{2}$ (Line 2834). As such, we add $C_{1}^{s}$ and $C_{2}^{s}$ after xor x $_{1}$ (Line 31-32). $C_{1}^{s}$ will be connected to $A$ and $C_{2}^{s}$ will be connected to $B$ (Line 33-34). The common subset of postsets will repeat in the resulting Petri net (Line 24-26). Subsequently, we can add two $\tau$ transitions representing $c s_{1}$ and $c s_{2}$ (Line 2). These two transitions are mutually exclusive (as shown below in Figure 5), such that the chosen path after xor 1 can be determined by the case currently being executed. That is, $C_{1}^{s}$ may only fire with a token in $x o r_{1}$ and after $c s_{1}$ has fired. This way, the execution of $A$ is specific to case $c s_{1}$, whereas the execution of $B$ is specific to case $c s_{2}$.

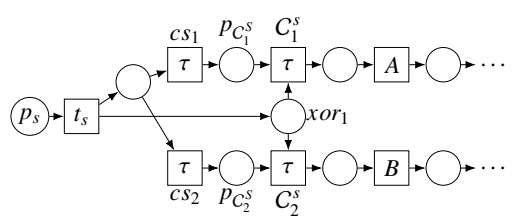

Fig. 5: Enforcing cases in the merged Petri net (splits).

In case of common transitions with different presets, a similar procedure is required to ensure that all incoming conditions are satisfied (Line 8-19). For example, we may have a similar rule in two cases, where in one case a certain

${ }^{1}$ Alternatively, a common prefix can be computed, from where every subsequent difference between the input Petri nets is resolved by a different branch. The resulting merged Petri net will effectively be a process tree, where the ends of all branches are joined together by a common end transition. This leads to an abundance of traces (i.e. the amount of traces equals the amount of cases), which would i) reduce understandability and maintainability and ii) increases the risk of inconsistencies when a transition needs to be changed (as a change of that transition is required for every occurrence of that transition in the process tree, which is potentially as much as the amount of cases). 
input condition is true at the initial state and, in the other case, that condition may be derived by another rule. Consequently, the inputs of that common transition may differ, requiring a synchronisation step to only proceed when all incoming transitions have fired. As such, we add a $\tau$ transition $C_{i}^{j}$ for each incoming path.

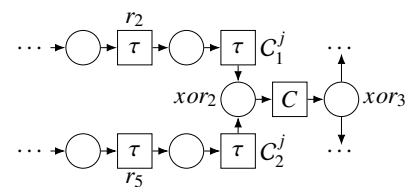

Fig. 6: Enforcing cases in the merged Petri net (joins).

The full resulting Petri net $N_{M}$ is represented in Figure 7. Note that the purpose of the $C^{s}$ transitions here is immediately clear, as the co-occurrence of $A$ and $D$ for case $c s_{1}$ (and $B$ and $E$ for case $c s_{2}$ ) is warranted. In Figure 7, the execution of the transitions in case $c s_{1}$ is highlighted in purple, while the auxiliary routing to preserve dependencies is highlighted in orange.

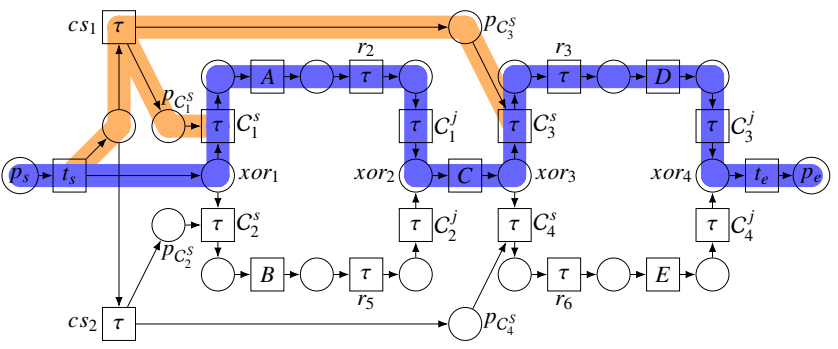

Fig. 7: Merged Petri net $N_{M}$ from $N_{1}$ and $N_{2}$.

The examples shown so far comprise different cases with distinctive paths that merge somewhere in the process. However, a situation where a transition occurring in one case is skipped in another case requires additional measures, as there is no immediate transition following the xor (as it is skipped). As such, certain $C^{s}$ transitions may end up without outgoing arcs. This problem can be illustrated with two cases: Case $c s_{1}=\left\{r_{1}: \Rightarrow A, r_{2}: A \Rightarrow B, r_{3}: B \Rightarrow D\right\}$ and Case $c s_{2}=\left\{r_{4}: \Rightarrow A, r_{5}: A \Rightarrow B, r_{6}: A \Rightarrow C\right.$, $\left.r_{7}: B \Rightarrow D, r_{8}: B \Rightarrow E\right\}$, resulting in traces $\langle A, B, D\rangle$ and $\langle A, B, C, D, E\rangle$ or $\langle A, C, B, D, E\rangle$ respectively, as shown graphically in Figure 8. A merge of both Petri nets would need to comprise an optional concurrent execution of $C$ with $B$ and an optional concurrent execution of $E$ with $D$, as $C$ and $E$ only occur in $c s_{2}$ and not in $c s_{1}$.

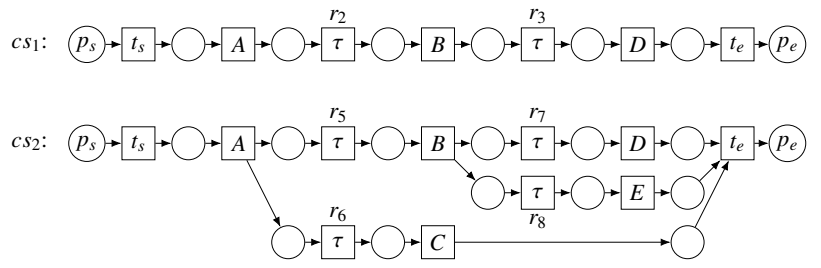

Fig. 8: Example resulting in a skipped transition $(C$ and $E)$.

Using the basic principles outlined above, however, the corresponding Petri net $N_{M}$ would be generated as shown in Figure 9. Note that there are three transitions (indicated with a light gray background with dashed borders) that are not fully connected: $C_{2}^{s}$ and $C_{4}^{s}$ have an empty postset, whereas $C_{2}^{j}$ has

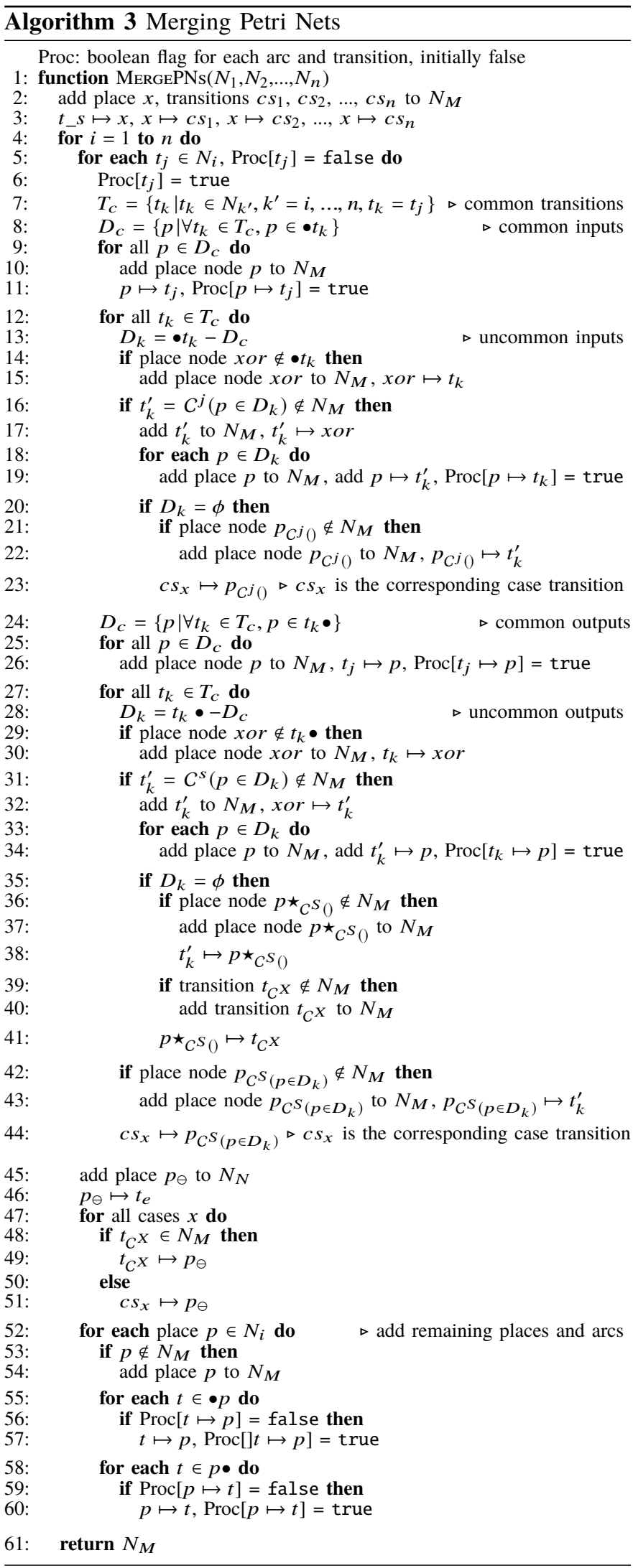

an empty preset. This is a result of the skipped transitions $C$ and $E$ that are concurrent with some other transitions that are common between both cases.

This is resolved by adding a set of $p \star_{C^{s}}$ places to the postset of the affected $C^{s}$ transitions (Line 35-38), which are synchronised by transition $t_{C^{X}}$ into place $p_{\ominus}$ (Line 39- 


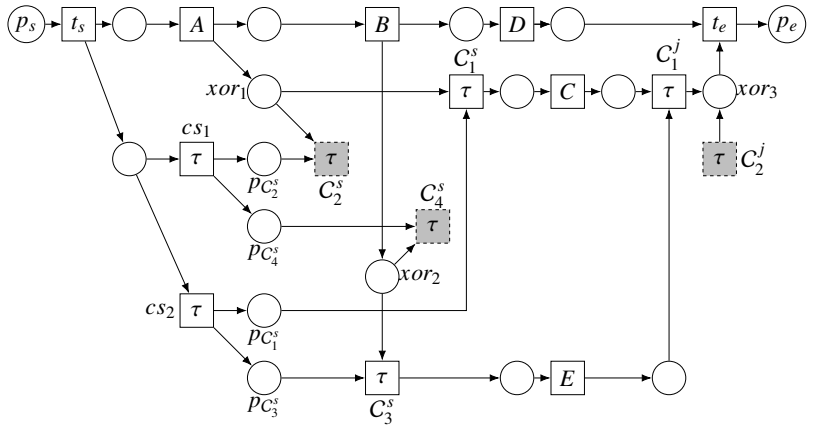

Fig. 9: Missing connections with skipped transitions.

51). Additionally, a place $p_{C^{j}}$ is added to the preset of the unconnected $C^{j}$ transitions (Line 20-23). The required additions are highlighted in purple in Figure 10.

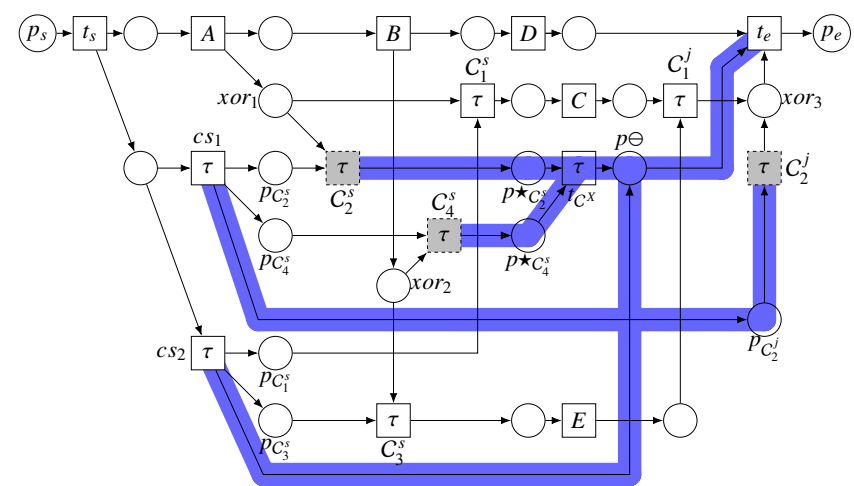

Fig. 10: Additional connections to support skipped transitions.

Finally, after all Petri nets $N_{i}$ are merged, we optimise the merged Petri net $N_{M}$ by removing all redundant $\tau$ transitions using the reduction techniques described in [16], which preserve the properties of liveness and boundedness. That is, if $\left(N_{M}, M_{0}\right)$ is live and 1-bounded, then the reduced net $\left(N_{M}^{\prime}, M_{0}^{\prime}\right)$ is live and 1-bounded as well [16]. As the reduction only removes $\tau$ transitions, $N_{M}^{\prime}$ is behaviourally equivalent to $N_{M}$. The resulting optimised merged Petri net of $N_{M}$ in Figure 7 is depicted graphically in Figure 11.

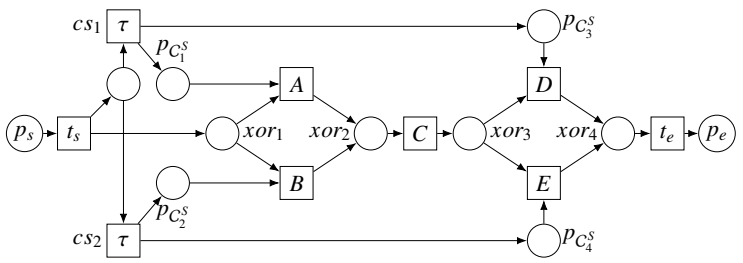

Fig. 11: Optimised Petri net $N_{M}$ from Fig. 7.

\section{Correctness properties}

The Petri net $N$ obtained from Alg. 2 represents exactly the behaviour as specified by a case-specific rule set $R_{C}$. That is, there exist no traces in $N$ that are not specified by $R_{C}$, and there is no behaviour allowed by the rules in $R_{C}$ that is not allowed by $N$. This property can be summarised as follows:

Lemma 1: Let $R_{C}$ be a rule set, and $N$ be the Petri net obtained by applying Alg. 2 to $R_{C}$. Then, there is a derivation $L_{1}, \ldots, L_{n}$ in $R_{C}$ iff there exists a sequence of markings $m_{0}, m_{1}, \ldots, m_{n^{\prime}}$ in $N$ obtained through firing transitions $t \_L_{1}, \ldots, t \_L_{n}$, where $m_{i}$ is reachable from $m_{i-1}$ for $i \in\left\{1, \ldots, n^{\prime}\right\}$.

Proof. See Appendix A.

The merged Petri net merges the behaviour of the input nets, such that it allows only those traces that are allowed by one of its input nets. As such, it constitutes a correct merge of the behaviour of the input nets, which can be formulated formally as follows:

Lemma 2: Let $N_{I N}$ be the set of input Petri nets to Alg. 3 (as obtained from Alg. 2), and $N_{M}$ be the merged Petri net obtained by applying Alg. 3 to $N_{I N}$. Then, there is a trace $t_{-} L_{1}, \ldots, t_{-} L_{n}$ in $N_{M}$ iff there exists a trace $t_{-} L_{1}, \ldots, t L_{-} L_{n}$ in some $N_{i} \in N_{I N}$.

Proof. See Appendix A.

In addition to the behavioural properties, the generated Petri nets satisfy a number of important structural properties, irrespective of the process to be supported. First of all, each place in the merged Petri net $N_{M}$ contains at most 1 token for every possible reachable marking. This property can be formulated as follows:

Lemma 3: Let $R$ be a rule set, and $N_{M}$ be the Petri net obtained by applying Alg. 1-3 to $R$. The merged Petri net $N_{M}$ is 1-bounded.

Proof. See Appendix A.

Finally, for every reachable marking (that is at any state) there is an enabled transition that can fire. As such, the merged Petri net $N_{M}$ is constructed in such a way that there exist no deadlocks and any possible execution can finish, irrespective of the path chosen:

Lemma 4: Let $R$ be a rule set, and $N_{M}$ be the Petri net obtained by applying Alg. 1-3 to $R$. The merged Petri net $N_{M}$ is $L_{1}$-live and deadlock-free.

Proof. See Appendix A.

As such, Alg. 1-3 produce a correct and sound Petri net $N_{M}$ from rule set $R$. That is, the relation between the behaviour allowed by $N_{M}$ and the behaviour allowed by the rules in $R$ is bijective and $N_{M}$ is bounded, live and deadlock-free.

Theorem 1: Let $R$ be a rule set and $N_{M}$ be the Petri net obtained by applying Alg. 1-3 to $R . N_{M}$ is a correct and sound representation of the rules in $R$.

Proof. Follows directly from Lemma 1-4.

\section{E. Computation Complexity}

To show that the method presented above does not have any tractability issues, we provide a formal discussion of the computational complexity of each proposed algorithm.

Given a finite, modal defeasible theory $D$, the size $\Sigma$ of $D$ is the number of occurrences of literals plus the number of the rules in $D$. For each literal, we implement a hash table with pointers to rules where the literal occurs in; thus, retrieving the set of rules containing a given literal requires constant time.

Lemma 5: Algorithm 1 terminates and its computational complexity is $O\left(\Sigma^{2}\right)$.

Proof. $\Sigma$ is finite thus the cycles at Line 2 and 6 always end. The main and nested cycles examine at most $\Sigma$ rules / literals, respectively, while removal (Line 4) and deletion (Line 9) require constant time. 
Lemma 6: Algorithm 2 terminates and its computational complexity is $O\left(\Sigma^{2}\right)$.

Proof. Termination: same as before. Complexity: the main cycle (Line 9-30) is iterated at most $\Sigma$ times, and so are the nested ones. The cycle at Line $22-26$ produces 8 new symbols for each rule in $R^{\prime} \in O(\Sigma)$, and thus $8 \cdot \Sigma \in O(\Sigma)$. As such, the complexity of the outer cycle is $O\left(\Sigma^{2}\right)$. All assignment operations and controls can be performed in constant time.

Theorem 2: Algorithm 3 is in $O\left(\Sigma^{4}\right)$. The overall complexity of Algorithm 2 and Algorithm 3 is $O\left(\Sigma^{2}\right)+O\left(\Sigma^{4}\right)=O\left(\Sigma^{4}\right)$.

Proof. Termination: trivial. There are no repeat-until/while cycles. Each for cycle iterates at most $\Sigma$ times, with $\Sigma$ finite. This also proves the complexity. (Note that structures such as the one at Line 7 of Algorithm 3 can be computed in linear time given the implementation proposed.)

\section{Evaluation}

We implemented our approach in a tool called Rules2PetriNet ${ }^{2}$ to assess the performance of the approach as well as the quality of the generated Petri nets. The tool takes as input a set of cases, each containing a set of rules. Its output is a merged Petri net $N_{M}$ that represents the behaviour as specified by all rules in the input cases.

Using this tool, we conducted a two-pronged (quantitative and qualitative) evaluation. First, we executed a quantitative evaluation assessing the performance and correctness of our approach compared to a state-of-the-art technique, using a large set of synthetic models. Second we performed a qualitative evaluation of our method on a real-life case containing the rules of a customer support process, to show the feasibility of our approach in a real-life setting.

All performance tests were executed on a machine with a quad core Intel ${ }^{\circledR}$ Core $^{\mathrm{TM}}$ i5-5300U $2.3 \mathrm{GHz}$ with $8 \mathrm{~GB}$ memory, running Linux 14.04. To eliminate load times, we executed each test five times and recorded average time of three executions, removing the fastest and the slowest execution.

\section{A. Quantitative evaluation with synthetic models}

First, we created a set of Petri nets, each representing an input scenario from which we generated all cases and their corresponding rules. As such, we obtain rules for these different scenarios. This allowed us to have a structured set of input scenarios with an increasing complexity. The 21 synthetic Petri nets comprise increasing branch counts and branch lengths for both and-splits and xor-splits. Furthermore, the input models contain multiple successive and-constructs or xor-constructs, to maximise the amount of cases and, therefore, potential computational complexity.

We assessed the performance of our tool by generating a merged Petri net for each input scenario. Subsequently, we assessed the quality of the generated Petri nets in terms of the amount of nodes and compared the resulting Petri net with the input Petri net to check behavioural equivalence.

To the best of our knowledge, there exist no tools that are able to transform regulations into Petri nets while preserving case-specific dependencies among subsequent exclusive branches. However, an alternative approach could be

${ }^{2}$ https://bitbucket.csiro.au/projects/BPLI/repos/rules2petrinet/ to generate all derivations from the rules and generate the Petri nets from the derivations. The Inductive Miner (IM) plugin [18] in the ProM 6.5.1 environment [19] is able to obtain a Petri net from an event log, which contains a set of traces (or derivations) that each consist of the sequence of events produced by a case [20]. As the approach of [18] requires an event log as input (instead of a set of rules), we generated an event $\log$ for each input net using the approach presented in [21], to simulate a scenario where we would have a set of derivations from rules.

Subsequently, we generated a Petri net from the event log (using IM) and assessed the performance in comparison with our approach. The evaluation approach for each scenario is shown graphically in Figure 12.

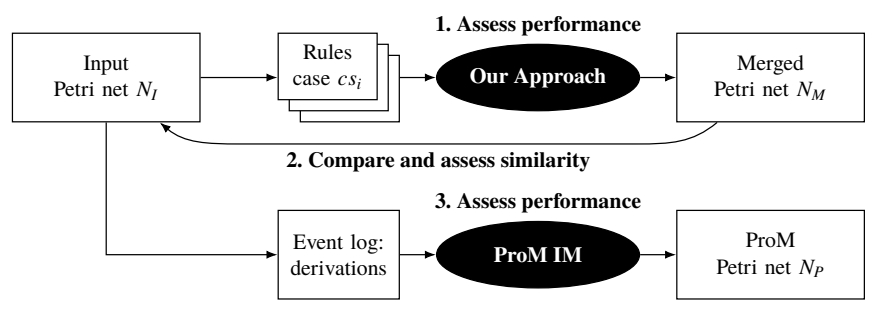

Fig. 12: Evaluation method for each scenario.

Table I provides an overview of the details of each synthetic Petri net. The two rightmost columns show the performance of our approach (i.e. Alg.1-3 and the optimisation) and ProM IM respectively.

For our approach, xor-branches naturally result in higher complexity, because they constitute different cases and are, therefore, represented by different case-specific Petri nets that need to be merged. Although concurrency combinatorially increases the amount of derivations, they have very little effect on the execution times for our approach, as they represent a single case and Petri nets are designed to model concurrent systems (hence interleaving is not an issue). For ProM IM, however, the amount of distinct traces (i.e. derivations) determines the execution time.

For each scenario, our approach outperforms ProM IM, with the exception of scenario 21 (42.85s vs 39.68s). Contrary to our approach, however, ProM IM provides a generalised Petri net that does not take into account dependencies among consecutive xor-constructs. As such, the resulting Petri net from ProM IM allows behaviour that is not allowed according to the rules and can, as a result, not be guaranteed to be compliant with the rules. In our approach, on the other hand, these dependencies are taken into account by default and can be removed automatically (and, therefore, generalised) when required. Consequently, for very large models the performance of our approach is comparable with ProM IM, as we are specifying consecutive branching constraints in the model as well, which naturally results in a larger number of transitions in case of our approach. Therefore, a direct comparison of the amount of nodes in both models is pointless.

There were three scenarios where ProM IM could not finish within 90s due to the large number of derivations. The large number of derivations in case of complex concurrent models (scenario 1 to 4 ) shows the infeasibility of generating all derivations prior to constructing the required model. 
TABLE I: Details of synthetic scenarios.

\begin{tabular}{|c|c|c|c|c|c|c|c|c|c|c|c|c|c|}
\hline Id & and-constr. & and-br & xor-constr. & $x o r-b r$ & Act. & Rules & Cases & Trans. & Places & Arcs & Derivations & Our approach (s) & ProM IM (s) \\
\hline 1 & 7 & 4 & 0 & 0 & 148 & 148 & 1 & 150 & 172 & 342 & $8.21 \cdot 10^{8}$ & 0.01 & Timed out \\
\hline 2 & 1 & 16 & 0 & 0 & 322 & 322 & 1 & 324 & 340 & 678 & $6.88 \cdot 10^{369}$ & 0.02 & Timed out \\
\hline 3 & 16 & 2 & 5 & 4 & 164 & 176 & 16 & 182 & 189 & 433 & 24,192 & 0.03 & 62.40 \\
\hline 4 & 32 & 2 & 9 & $8 \times 4+1 \times 8$ & 328 & 352 & 32 & 362 & 373 & 861 & 48.384 & 0.09 & Timed out \\
\hline 5 & 0 & 0 & 1 & 8 & 162 & 169 & 8 & 164 & 159 & 337 & 8 & 0.02 & 0.50 \\
\hline 6 & 0 & 0 & 1 & 16 & 322 & 337 & 16 & 324 & 311 & 665 & 16 & 0.05 & 0.57 \\
\hline 7 & 0 & 0 & 4 & 2 & 42 & 49 & 16 & 72 & 65 & 207 & 16 & 0.01 & 0.66 \\
\hline 8 & 0 & 0 & 5 & 2 & 52 & 61 & 32 & 102 & 81 & 351 & 32 & 0.02 & 0.78 \\
\hline 9 & 0 & 0 & 6 & 2 & 62 & 73 & 64 & 148 & 97 & 639 & 64 & 0.04 & 0.97 \\
\hline 10 & 0 & 0 & 7 & 2 & 72 & 85 & 128 & 226 & 113 & 1,247 & 128 & 0.08 & 1.14 \\
\hline 11 & 0 & 0 & 8 & 2 & 82 & 97 & 256 & 368 & 129 & 2,559 & 256 & 0.19 & 1.44 \\
\hline 12 & 0 & 0 & 9 & 2 & 92 & 109 & 512 & 638 & 145 & 5,407 & 512 & 0.46 & 2.39 \\
\hline 13 & 0 & 0 & 10 & 2 & 102 & 121 & 1,024 & 1,164 & 161 & 11,583 & 1,024 & 1.11 & 3.70 \\
\hline 14 & 0 & 0 & 11 & 2 & 112 & 133 & 2,048 & 2,202 & 177 & 24,927 & 2,048 & 2.72 & 7.60 \\
\hline 15 & 0 & 0 & 12 & 2 & 122 & 145 & 4,096 & 4,264 & 193 & 53,631 & 4,096 & 6.78 & 17.77 \\
\hline 16 & 0 & 0 & 13 & 2 & 132 & 157 & 8,192 & 8,374 & 209 & 115,103 & 8,192 & 18.64 & 40.91 \\
\hline 17 & 0 & 0 & 14 & 2 & 142 & 169 & 16,384 & 16,580 & 225 & 246,207 & 16,384 & 73.29 & 79.50 \\
\hline 18 & 0 & 0 & 4 & 4 & 82 & 121 & 256 & 388 & 147 & 1,597 & 256 & 0.12 & 1.03 \\
\hline 19 & 0 & 0 & 5 & 4 & 102 & 153 & 1,024 & 1,192 & 187 & 6,549 & 1,024 & 0.69 & 2.31 \\
\hline 20 & 0 & 0 & 6 & 4 & 122 & 185 & 4,096 & 4,300 & 227 & 29,165 & 4,096 & 4.10 & 8.97 \\
\hline 21 & 0 & 0 & 7 & 4 & 142 & 217 & 16,384 & 16,624 & 267 & 131,653 & 16,384 & 42.85 & 39.68 \\
\hline
\end{tabular}

However, the most complicated scenario (17) with 14 consecutive $x o r$-splits with 2 branches $(16,384$ cases in total and 246,207 arcs) still took only 73.3 seconds to complete, while scenario 21 with 7 consecutive $x o r$-splits with 4 branches $(16,384$ cases in total and 131,653 arcs) took 42.9 seconds. The performance of both scenarios is still acceptable, particularly considering that model generation is a design-time exercise. For less complicated scenarios (and more realistic ones), the execution time is measured in milliseconds. This implies that the method could even be applied in a runtime setting, where on-the-fly reconfiguration is required.

Finally, the resulting Petri nets were compared to the original input Petri nets. The traces of each pair of nets (i.e. input vs. resulting) were compared. Each of the scenarios showed a one-on-one match, which implies that all of the produced nets were behaviourally equivalent to their respective input nets.

\section{B. Qualitative evaluation with a real-life process}

For the qualitative evaluation, we used a real-life process concerning a customer support process in a telecom company in Australia, as presented in [22]. The process contains 24 visible activities, $8 \tau$ transitions and a start and end transition. The normative model is shown graphically in Figure 13.

Again, we derived the cases and corresponding rules from this process, that will be used as input for our tool. This resulted in 92 different cases having on average 19 rules, with the smallest case containing 4 rules and the largest case containing 24 rules. In total, the process contains 33 distinct rules among all cases. Subsequently, the 92 different casespecific Petri nets were obtained using Alg. 2. By using Alg. 3, these individual Petri nets were merged to obtain Petri net $N_{M}$.

The resulting net $N_{M}$ contains 24 transitions representing task literals, 92 case-specific transitions and 18 additional silent transitions $\left(C^{s}\right.$ and $\left.C^{j}\right)$. As the successive xor-splits in the customer support process are independent in this process (there are no case-specific dependencies among xor-splits), the case-specific transitions can be removed along with the additional $C^{s}$ and $C^{j}$ transitions for which the preset and postset equals 1 . This results in a total of 42 remaining transitions. An overview of the results is shown in Table II, where the details of the full model (i.e. including cases) are

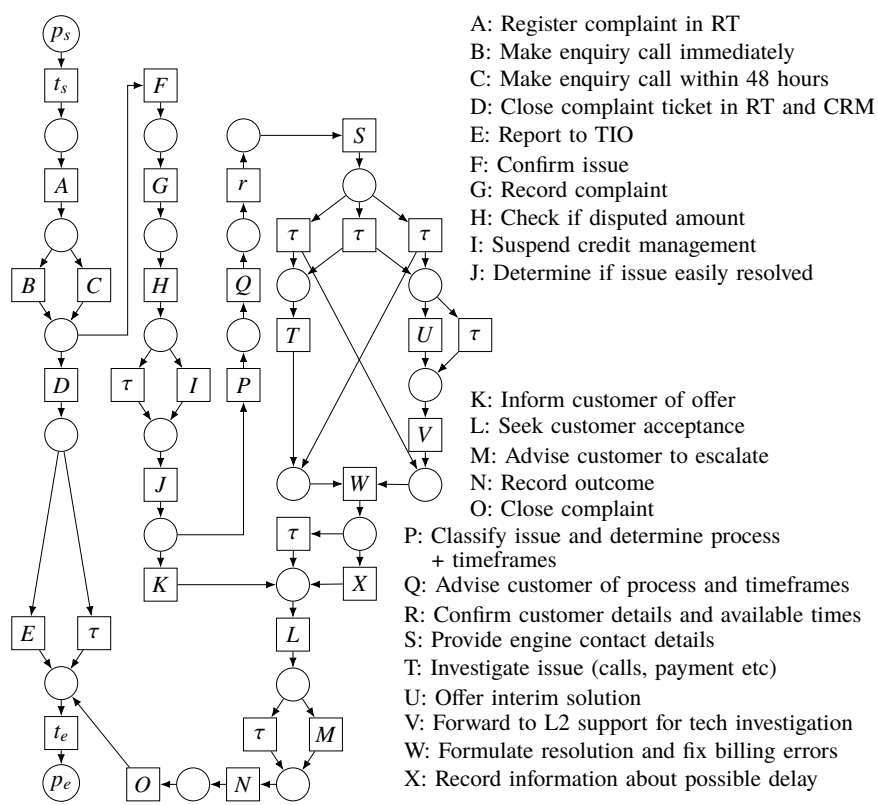

Fig. 13: Customer support process [22].

shown between parentheses. The optimised resulting net is sound and behaviourally equivalent to the initial input process, as verified using the approach explained in Section V-A.

TABLE II: Performance of the customer support process.

\begin{tabular}{cccccc|cc}
\hline Act. & Rules & Cases & Trans. & Places & Arcs & Our approach & ProM \\
\hline 24 & 33 & 92 & $42(134)$ & $37(63)$ & $92(934)$ & $0.080 \mathrm{~s}$ & $1.090 \mathrm{~s}$ \\
\hline
\end{tabular}

Consequently, for more a structurally complicated real-life process, the algorithms presented in this paper have shown to be very well able to generate a correct and sound process from a set of rules and is performing well $(80 \mathrm{~ms})$ and considerably faster than ProM IM (1.090s).

\section{Related Work}

The approach we presented departs from the standard BDI architecture and agent programming languages implementing it (e.g., 3APL-2APL [23][24], JASON [25]), and extensions with norms in several respects (e.g., BOID by [26], while we refer the reader to [27] for an overview). While in these 
approaches the agent has to select (partially) predefined plans from a plan library, we propose on-the-fly business process generation (corresponding to a set of plans) to meet the objectives without violating the norms it is subject to.

In [28], Alechina et al. present a BDI-based agent programming language based on 2APL for norm-aware agents; a normaware agent can deliberate on its goals, norms, and sanctions before deciding which plan to select and execute. Our agents are de facto norm-aware. A major issue of this work is that if a goal triggers two (or more) sanctions, each of which is lower in rank than the achievement of that goal, the agent will try to achieve that particular goal even if the sum of the two sanctions is higher in rank.

Automated planning is a technique to organise actions with the aim of achieving some pre-specified goal starting from the current state of the system [13]. Each action features a set of preconditions that have to be satisfied prior to its execution and a set of effects, which specify the state change resulting from its execution. There are frameworks to generate plans (e.g., KPG [29] and Golog [30]), but these are typically based on classical AI planning and do not consider norms. In addition, many automated planning approaches in the business process domain focus on runtime adaptation of pre-specified processes, concerning runtime repair instead of design time process generation based on rules (see e.g. [5][6]). Automated planning techniques require a goal to be specified along with an initial state. However, in case of multiple initial states, multiple possible plans have to be generated (i.e. all possible traces for each existing input case), that need to be merged in order to represent a full business process [7]. As such, providing a business process model that supports all possible traces as specified by the rules remains a challenge.

DECLARE provides an approach for declarative specifications of business processes by means of constraints [31] and graphical representations are available to visualise the constraints and the activities in the model [32]. However, the graphical representation shows the exact constraints and does not provide an actual process model imposed by the rules (i.e. a model showing the possible traces). In [8], an approach is presented to convert a DECLARE model to a behaviourally equivalent Petri net. However, this approach leads to multiple transitions representing the same activity and, therefore, a highly complicated model. Our approach, on the other hand, provides a model without additional duplication of transitions. In [33], an extension is developed on top of BPMN, BPMN$\mathrm{D}$, which supports declarative process modelling. It allows to transform DECLARE models into readable BPMN-D models. This approach, however, does not support concurrency. Additionally, DECLARE is based on Linear Temporal Logic (LTL), which is not able to represent certain complex norms [34] and can, as such, not be used for this purpose.

Sardina et al. [35] provide an account of goals in the view of declarative aspects by integrating BDI failure mechanisms with Hierarchical Task Network (HTN) planning techniques. HTN planning is notoriously undecidable even if no variables are allowed, or PSPACE-hard if restrictions are given either on non-primitive tasks or on the ordering of tasks. The main feature of their $\mathrm{CAN}^{\mathcal{A}}$ is its detailed operational semantics where, if a plan fails, alternative plans for achieving the goal are tried. Compared to theirs, the approach presented in this paper has the advantage that we generate all possible plans compactly at design time.

Finally, in [36][37], a declarative approach is used to create compliant business processes on the fly using BPMN notation. Although their approach has been proven sound, a formal proof of completeness is lacking. Moreover, our choice of using Petri nets instead of BPMN notation facilitates us to prove the formal verification of soundness and completeness.

\section{Conclusion}

Enterprises spend a significant effort to maintain their business processes to be efficient and compliant with the regulations affecting their operational environment. Declarative formalisms are able to describe both the means an organisation uses to reach its business objectives and the regulations impacting the organisational environment. Although extremely important, it is difficult to adhere to such rules, as the amount of rules is usually very large. In addition, understandability of such large sets of rules is generally very low.

This paper addresses these problems by presenting a new method to automatically and efficiently transform a set of business rules and regulations to a behaviourally equivalent corresponding Petri net. As such, this paper presents advanced formal, complete, automated algorithms to build a compliant by design business process starting from such declarative specifications. The approach presented in this paper is particularly useful for organisations as it ensures that their business processes are compliant by design and resilient to changes in regulations or corporate policies. More specifically, rule changes can be automatically adopted in the design of the affected business processes and their effect on the actual process flow can be immediately visualised.

We implemented the approach in a tool and subsequently evaluated the approach on a set of synthetic scenarios and a real-life process, to assess the performance and the correctness of the resulting Petri net. The results show that the algorithms perform very well, even for highly complex scenarios with 16,384 cases. Furthermore, in almost all scenarios, our approach significantly outperforms a state-of-the-art technique for process synthesis from event logs.

The resulting Petri nets provide an exact representation of the behaviour as specified by the rules and serve, therefore, as an accurate visualisation of those rules. The application to the real-life process showed that the approach is feasible in real-life scenarios and produces an understandable Petri net.

In this paper, loops are not considered in the rules. Although the rules themselves allow to specify loops, the use of derivations prevented us from including such behaviour in the final model. Furthermore, the coverage of the applicability of the resulting Petri net is limited to the cases explicitly given by the designers. However, the constructions could be used at runtime to generate a Petri net corresponding to a set of rules and an input case on the fly. Additionally, future lines of research will lead us to an elaborate empirical evaluation to study the understandability and usability of the resulting Petri net compared to the rule set as perceived by end users.

\section{Appendix: Complete Proofs}

Lemma 1: Let $R_{C}$ be a rule set, and $N$ be the Petri net obtained by applying Algorithm 2 to $R_{C}$. Then, there is 
a derivation $L_{1}, \ldots, L_{n}$ in $R_{C}$ iff there exists a sequence of markings $m_{0}, m_{1}, \ldots, m_{n^{\prime}}$ in $N$ obtained through firing transitions $t \_L_{1}, \ldots, t \_L_{n}$, where $m_{i}$ is reachable from $m_{i-1}$ for $i \in\left\{1, \ldots, n^{\prime}\right\}$.

Proof. Proof of only if part by induction:

Base case: When $n=1$, we have the derivation $L_{1}$ which means that there is a rule $r: l_{1}, \ldots, l_{n} \Rightarrow L_{1}$ where all $l_{i} \mathrm{~s}$, $i \in\{1, \ldots, n\}$ are true in the initial state. In the preprocessing step (Algorithm 1), these literals are deleted from the body of the rule and since the body is empty (Line 10-11), there will be arcs $t_{-} s \mapsto \mapsto t_{-} L_{1}: t_{-} s \mapsto p \mapsto t_{-} L_{1}$. Considering markings $m_{0}=\left\{p_{\_} s\right\}, m_{1}=\{p\}$, the sequence $m_{0}, m_{1}$ is the marking sequence that fires $t_{-} L_{1}$.

Induction step: We assume that for a derivation $L_{1}, \ldots, L_{k}$, there is a sequence of markings $m_{0}, m_{1}, \ldots, m_{k^{\prime}}$ that fires transitions $t_{-} L_{1}, \ldots, t_{-} L_{k}$ and we show that for a derivation $L_{1}, \ldots, L_{k}, L_{k+1}$ there is a sequence of markings $m_{0}, \ldots, m_{k^{\prime}}, \ldots, m_{k^{\prime \prime}}$ that fires transitions $t_{-} L_{1}, \ldots, t_{-} L_{k}, t_{-} L_{k+1}$. When we have a derivation $L_{1}, \ldots, L_{k}, L_{k+1}$, it means that at the last step of the derivation we use a rule $r: l_{1}, \ldots, l_{n} \Rightarrow L_{k+1}$ where $l_{1}, \ldots, l_{n}$ are in $\left\{L_{1}, \ldots, L_{k}\right\}$. According to Algorithm 2 (Line 14-15), we have $\operatorname{arcs} t_{-} l_{i} \mapsto \mapsto t_{-} r: t_{-} l_{i} \mapsto p_{i} \mapsto t \_r$. By the induction assumption, all transitions $t_{-} l_{i}$ have fired; we thus have a token in each place $p_{i}$ in $m_{k^{\prime}}$. This implies $t_{-} r$ can fire. There are two cases in the algorithm based on the number of rules with head $L_{k+1}$. If there is only a single rule (Line 1617), we have arcs $t \_r \mapsto \mapsto t L_{k+1}: t \_r \mapsto p^{\prime} \mapsto t \_L_{k+1}$. This means marking sequence $m_{0}, \ldots, m_{k^{\prime}}, m_{k^{\prime \prime}}$ where $m_{k^{\prime \prime}}=m_{k^{\prime}}-$ $\left\{p_{1}, \ldots, p_{n}\right\}+\left\{p^{\prime}\right\}$ can fire $t_{-} L_{k+1}$ and this proves the induction step. In the case that we have several rules $R_{C}^{\prime}=\left\{r_{1}, \ldots, r_{m}\right\}$ with head $L_{k+1}$ where $r_{i}: l_{1}^{i}, \ldots, l_{n}^{i} \Rightarrow L_{k+1}$ and $r=r_{y} \in R_{C}^{\prime}$ (Line 18-26), besides the arcs $t_{-} l_{j}^{i} \mapsto p_{j}^{i} \mapsto t_{-} r$, we also have arcs $t_{-} r_{i} \mapsto x_{i} \mapsto t_{-} r_{i}^{\prime} \mapsto p_{x} \mapsto t_{-} L_{k+1}$ and also $t_{-} s \mapsto p_{\oplus} \mapsto t_{-} r_{i}^{\prime}$. As we argued in the previous case, marking $m_{k^{\prime}}$ can fire $t_{-} r=t_{-} r_{y}$ and leave a token in place $x_{y}$. In addition, since $t_{-} s$ has fired, we have a token in place $p_{\oplus}$. These places are the only input places of transition $t \_r_{y}^{\prime}$ so it can fire. The marking sequence $m_{1}, \ldots, m_{k^{\prime}}, m_{k_{1}^{\prime \prime}}, m_{k_{2}^{\prime \prime}}$ where $m_{k_{1}^{\prime \prime}}=m_{k}^{\prime}-\left\{p_{1}^{y}, \ldots, p_{n}^{y}\right\}+\left\{x_{y}\right\}, m_{k_{2}^{\prime \prime}}=m_{k_{1}^{\prime \prime}}-\left\{x_{y}, p_{\oplus}\right\}^{2}+\left\{p_{x}\right\}$ can fire $t_{-} L_{k+1}$, which proves the induction step.

Proof of if part by induction:

Base case: Suppose there is a sequence of markings $m_{0}, m_{1}, \ldots, m_{n^{\prime}}$ that fires transition $t_{-} L_{1}$. According to Algorithm 2 , every transition for literals has exactly one input place (Line 11, 17 and 20). This means that there should be a token at input place $p$ of transition $t_{-} L_{1}$ in $m_{n^{\prime}}$. Since no other transition is fired, the only transition that can fire and put a token in this place is $t_{-} s$, meaning that we should have arcs $t_{-} s \mapsto p \mapsto t_{-} L_{1}$. We have these arcs only when we have a rule $r: l_{1}, \ldots, l_{n} \Rightarrow L_{1}$ where all $l_{i} \mathrm{~s}, i \in\{1, \ldots, n\}$ are true at the initial state. This means we have a derivation for $L_{1}$.

Induction step: We assume that for a sequence of markings $m_{0}, m_{1}, \ldots, m_{k^{\prime}}$ that fires transitions $t_{-} L_{1}, \ldots, t_{-} L_{k}$, there is a derivation $L_{1}, \ldots, L_{k}$ and we show that for a sequence of markings $m_{0}, \ldots, m_{k^{\prime}}, \ldots, m_{k^{\prime \prime}}$ that fires transitions $t \_L_{1}, \ldots, t \_L_{k}, t \_L_{k+1}$, there exists a derivation $L_{1}, \ldots, L_{k}, L_{k+1}$. For transition $t_{-} L_{k+1}$ to fire, there needs to be a token in the input place of $t_{-} L_{k+1}$ in marking $m_{k^{\prime \prime}}$. According to the algorithm, there are two cases where we can have a token at that place. In the first case (Line 17), we have arcs $t \_r \mapsto \mapsto t L_{k+1}: t_{-} r \mapsto p^{\prime} \mapsto t_{-} L_{k+1}$ and $p^{\prime}$ has only $t \_r$ as input which means $t \_r$ should have fired. Since $r: l_{1}, \ldots, l_{n} \Rightarrow L_{k+1}$ and according to the algorithm (Line 15), we have arcs $t_{-} l_{i} \mapsto \mapsto t_{-} r: t_{-} l_{i} \mapsto p_{i} \mapsto t_{-} r$, all $p_{i} \mathrm{~s}$ must have a token in some marking $m$ before $m_{k^{\prime \prime}}$, meaning that all $t_{-} l_{i} \mathrm{~s}$ are fired before. Since $t_{-} L_{1}, \ldots, t \_L_{k}$ are the only transitions fired, $\left\{l_{1}, \ldots, l_{n}\right\} \subseteq\left\{L_{1}, \ldots, L_{k}\right\}$. Therefore, by the induction assumption, we have a derivation for all $l_{i} \mathrm{~s}$ in body of rule $r$ and we can use this rule to derive $L_{k+1}$. We thus have a derivation $L_{1}, \ldots L_{k}, L_{k+1}$ and this proves the induction step. In the second case, we have arcs $t_{-} r_{i}^{\prime} \mapsto p_{x} \mapsto t_{-} L_{k+1}$ (Line 20 and 25) which means one of $t_{-} r_{i}^{\prime} \mathrm{s}$ should have fired to put a token in place $p_{x}$. All transitions $t_{-} r_{i}^{\prime} \mathrm{s}$ in the algorithm have two input places (Line 25-26): one coming from $t \_s$ $\left(t \_s \mapsto p_{\oplus} \mapsto t_{-} r_{i}^{\prime}\right)$ and one from the corresponding $t_{-} r_{i}$ $\left(t_{-} r_{i} \mapsto x_{i} \mapsto t_{-} r_{i}^{\prime}\right)$. Since $t_{-} s$ fires at the first step, place $p_{\oplus}$ always has a token and there needs to be a token at place $x_{i}$ for $t_{-} r_{i}^{\prime}$ to fire. Accordingly, $t_{-} r_{i}$ must have fired and we can use the same argument of the first case to prove that we have derivations for all $l_{j} \mathrm{~s}$ in body of rule $r_{i}$ and we can use this rule to derive $L_{k+1}$. Therefore we have a derivation $L_{1}, \ldots, L_{k}, L_{k+1}$, which proves the induction step.

Lemma 2: Let $N_{I N}$ be the set of Petri nets obtained through Algorithm 2, and $N_{M}$ be the merged Petri net obtained by applying Algorithm 3 to $N_{I N}$. Then, there is a trace $t_{-} L_{1}, \ldots, t_{-} L_{n}$ in $N_{M}$ iff there exists a trace $t_{-} L_{1}, \ldots, t_{-} L_{n}$ in some $N_{i} \in N_{I N}$.

\section{Proof. Proof of if part by induction:}

Base case: If $L_{1}$ is a trace in $N_{i}$, then we have arcs $t_{-} s \mapsto$ $p \mapsto t \_L_{1}$ and markings sequence $\left\{m_{0}=\left\{p \_s\right\}, m_{1}=\{p\}\right\}$ fires $t \_L_{1}$ (Line 11, Alg. 2). If $\bullet t_{-} L_{1}$ is the same $\forall N_{j} \in N_{I N}$ that contain $t_{-} L_{1}$, we will have two cases depending on $t_{-} s \bullet$ in $N_{j}$. In the first case, we have arcs $t_{-} s \mapsto p \mapsto t_{-} L_{1}$ in $N_{M}$ so the same marking sequence $\left\{m_{0}, m_{1}\right\}$ will fire $t_{-} L_{1}$ (Line 24-26, Alg. 3). In the second case, we have arcs $t \_s \mapsto x o r, x o r \mapsto C^{s}$, and $C^{s} \mapsto p \mapsto t_{-} L_{1}$ along with arcs $t_{-} s \mapsto x^{\prime} r^{\prime} \mapsto c s_{1} \mapsto p^{\prime} \mapsto C^{s}$ (Line 28-34, Alg. 3). Therefore, marking sequence $\left\{m_{0}=\left\{p \_s\right\}, m_{1}=\right.$ $\left.\left\{x o r, x o r^{\prime}\right\}, m_{2}=\left\{x o r, p^{\prime}\right\}, m_{3}=\{p\}\right\}$ fires $t_{-} L_{1}$. If $\bullet t_{-} L_{1}$ is different in one or more $N_{j} \in N_{I N}$ that contains $t_{-} L_{1}$ (Line 13-19, Alg. 3), we will also have two cases based on $t_{-} s \bullet$. In the first case, we have arcs $t_{-} s \mapsto p \mapsto C^{j} \mapsto x o r \mapsto t_{-} L_{1}$ so the mapping sequence $\left\{m_{0}=\left\{p_{-} s\right\}, m_{1}=\{p\}, m_{2}=\{x o r\}\right\}$ will fire $t_{-} L_{1}$. In the second case, we have arcs $t_{-} s \mapsto x o r_{1}$, xor $_{1} \mapsto C^{s}, C^{s} \mapsto p, p \mapsto C^{j}, C^{j} \mapsto$ xor $_{2} \mapsto t_{-} L_{1}$ along with arcs $t \_s \mapsto x o r^{\prime} \mapsto c s_{1} \mapsto p^{\prime} \mapsto C^{s}$. Therefore, marking sequence $\left\{m_{0}=\left\{p_{\_} s\right\}, m_{1}=\left\{x_{0}, x_{1}\right.\right.$, or $\left.^{\prime}\right\}, m_{2}=$ $\left.\left\{x_{0} r_{1}, p^{\prime}\right\}, m_{3}=\{p\}, m_{4}=\left\{x_{0} r_{2}\right\}\right\}$ fires $t_{-} L_{1}$.

Induction step: we assume that for a trace $t_{-} L_{1}, \ldots, t_{-} L_{k}$ in $N_{i}$, we have a trace $t_{-} L_{1}, \ldots, t_{-} L_{k}$ in $N_{M}$. Then we show that for a trace $t_{-} L_{1}, \ldots, t_{-} L_{k}, t_{-} L_{k+1}$ in $N_{i}$, we will have a trace $t_{-} L_{1}, \ldots, t L_{k}, L_{-} L_{k+1}$ in $N_{M}$. If $t \_L_{1}, \ldots, t \_L_{k}, t \_L_{k+1}$ is a trace in $N_{i}$, then for $t_{-} L_{k+1}$ to fire, there exists a token in the input place of $t_{-} L_{k+1}$ in $N_{i}$. This place will have a token if there exists a rule $r: l_{1}, \ldots, l_{n} \Rightarrow L_{k+1}$ where all $l_{m} \in\left\{l_{1}, \ldots, l_{n}\right\}$ are among $L_{1}, \ldots, L_{k}$ so that transition $t_{-} r$ will fire and lead a token to the input place of $t_{-} L_{k+1}$ directly or via transition $t \_r^{\prime}$. If $\bullet t_{-} L_{k+1}$ is the same in all Petri nets $N_{j} \in N_{I N}$ that contain $t_{-} L_{k+1}$, the same structure of $N_{i}$ exists in $N_{M}$ (Line 
8-11, Alg. 3). Therefore, the same arcs that lead a token from rule transition $t_{-} r$ to fire $t_{-} L_{k+1}$ will be in $N_{M}$. Based on the induction assumption, since $t_{-} L_{1}, \ldots, t_{-} L_{k}$ is a trace in $N_{M}$, transition $t_{-} r$ and consequently $t_{-} L_{k+1}$ can fire in $N_{M}$, that is $t_{-} L_{1}, \ldots, t_{-} L_{k}, t_{-} L_{k+1}$ is a trace in $N_{M}$. Note that the transitions for rules will be the same in $N_{M}$ as in $N_{I N}$, because their inputs and outputs are the same in all Petri nets. In the case that inputs of transition $t_{-} L_{k+1}$ are different in one or more Petri nets $N_{j} \in N_{I N}$ that contain $t_{-} L_{k+1}$ (Line 13-19, Alg. 3), there exist arcs from these different inputs to different transitions $C^{j}$. Therefore, we have arcs $p \mapsto C^{j}$ where $p$ is an input place of $t_{-} L_{k+1}$ in $N_{i}$, which is different from the input place of $t_{-} L_{k+1}$ in $N_{j}$. Additionally, we have $\operatorname{arcs} C^{j} \mapsto$ xor $\mapsto t_{-} L_{k+1}$. As argued before, place $p$ will have a token, because $L_{1}, \ldots, L_{k}$ is a trace in $N_{M}$ and transition $t_{-} r$ will fire and lead a token to this place. Consequently, $C^{j}$ will fire and so will $t_{-} L_{k+1}$.

Proof of only if part by induction:

Base case: If $L_{1}$ is a trace in $N_{M}$, it means we either have (1) $t \_s \mapsto p \mapsto t \_L_{1}$, or (2) $t \_s \mapsto x o r \mapsto C^{s} \mapsto p \mapsto t \_L_{1}$, or (3) $t_{-} s \mapsto p \mapsto C^{j} \mapsto x o r \mapsto t L_{-} L_{1}$, or (4) $t_{-} s \mapsto x o r \mapsto$ $C^{s} \mapsto p \mapsto C^{j} \mapsto x o r \mapsto t_{-} L_{1}$. In (1), $t_{-} L_{1}$ is a transition in all $N_{i}$ s and we have trace $L_{1}$ in all Petri nets. In (2), transition $C^{s}$ will have an input xor place from some of case transitions $c s_{x}$. Trace $L_{1}$ is a trace in all the corresponding Petri nets. In (3), $L_{1}$ is a trace in all Petri nets corresponding to the cases related to $C^{j}$ meaning all cases in which the input of $L_{1}$ is the same in their Petri nets as the inputs of $C^{j}$. Finally in (4), $L_{1}$ is a trace in all Petri nets corresponding to input cases of $C^{s}$ which are the same as corresponding cases for $C^{j}$.

Induction Step: We assume that for a trace $t_{-} L_{1}, \ldots, t_{-} L_{k}$ in $N_{M}$, we have a trace $t_{-} L_{1}, \ldots, t_{-} L_{k}$ in some $N_{i} \in N_{I N}$, then we show that for a trace $t_{-} L_{1}, \ldots, t_{-} L_{k}, t_{-} L_{k+1}$ in $N_{M}$, we will have a trace $t_{-} L_{1}, \ldots, t_{-} L_{k}, t_{-} L_{k+1}$ in some $N_{i} \in N_{I N}$. We prove this by contraposition, that is we show that if $L_{1}, \ldots, L_{k}, L_{k+1}$ is not a trace in any $N_{i} \in N_{I N}$, it will not be a trace in $N_{M}$. Based on the induction assumption, if $t_{-} L_{1}, \ldots, t_{-} L_{k}$ occurs in a trace in $N_{M}$, it will be a trace in some Petri nets $N_{i} \in N_{I N}$. If $t_{-} L_{1}, \ldots, t_{-} L_{k}, t_{-} L_{k+1}$ is not a trace in any $N_{i} \in N_{I N}$, then no rule is exists in the corresponding rule sets where $r: l_{1}, \ldots, l_{n} \Rightarrow L_{k+1}$ and all $l_{m} \in\left\{l_{1}, \ldots, l_{n}\right\}$ are among $L_{1}, \ldots, L_{k}$. Accordingly, there will be no path to lead a token to fire $t_{-} L_{k+1}$ in $N_{M}$ after trace $t_{-} L_{1}, \ldots, t L_{-} L_{k}$. This means $t_{-} L_{1}, \ldots, t_{-} L_{k}, t_{-} L_{k+1}$ is not a trace in $N_{M}$.

Lemma 3: Let $R$ be a rule set, and $N_{M}$ be the Petri net obtained by applying Algorithm 1-3 to $R$. The merged Petri net $N_{M}$ is 1 -bounded.

Proof. We prove by strong induction on the maximum number of transitions we need to traverse to reach a place.

Base case: When the maximum number of transitions needed to traverse to reach a place is 0 , we have only place $p_{s}$ which, by definition, has one token being a start place.

Induction step: We assume that for every place where the maximum number of transitions needed to traverse to reach is $k, k<n$, the place has at most one token and we prove that for a place where the maximum number of transitions needed to traverse to reach is $n$, it has at most one token. To prove this, we know that for places which have just one input, this is true. We consider places where $|\bullet p|>1$ and show that they can have at most one token. These places are:
1) When we have different rules with the same task as their heads, we have a place $p_{x}$ with more than one input from transitions $t_{-} r^{\prime}$ s (Line 25, Alg. 2). However, transitions $t \_r$ 's have a common input from place $p_{\oplus}$ and therefore only one of them can fire (Line 26, Alg. 2). This proves that place $p_{x}$ can have at most one token.

2) The other place with $|\bullet p|>1$ is $p_{\mathbf{m}}$, which has more than one input from transitions $t_{-} r^{\prime \prime}$ s (Line 30, Alg. 2). Since transitions $t \_r^{\prime \prime}$ s have a common input $p_{\sim}$, only one of them can fire and thus place $p_{\text {n }}$ can have at most one token.

3) The $x$ or places after $C^{j}$ transitions are the other places with more than one input (Line 17, Alg. 3). We have these places whenever the common transition in Petri nets have different inputs in those Petri nets. In this case, we have $\operatorname{arcs} C^{j} \mapsto x o r$ from different $C^{j}$ transitions. When the inputs of a transition $t$ are different in the Petri nets that we merged, this means there are different derivations in those Petri nets leading to $t$. This implies that there exists at least one common transition (which can be $t_{-} s$ ) in these Petri nets with different outputs. Based on the algorithm, we have an xor place after this transition along with arcs $t \mapsto x$ xor and xor $\mapsto C^{s}$ (Line 30-32, Alg. 3). Since one of the inputs of transitions $C^{s}$ is from the corresponding case transitions $c s_{x}$ (Line 43-44, Alg. 3) and only one of these transitions can fire (they share a common input $x$ ) (Line 3 , Alg. 3), we can have only one of these derivations in the merged Petri net. Accordingly, it is not possible for more than one transition $C^{j}$ to fire at the same time and, as a result, the xor place after them has at most one token.

4) The places $p_{C} s$ may also have more than one input (Line 44, Alg. 3). These inputs are from different case transitions and since only one of these transitions can fire at the same time, these places can have at most one token.

5) If the input places of a common transition in one Petri net are subset of the input of that transition in some other Petri net, we have places $p_{\ominus}$ which have more than one input (Line 4551, Alg. 3). The inputs of this place are from case transitions $c s_{x}$ or from transitions $t_{C^{X}}$ which, again, have inputs from $C^{s}$ transition with inputs from case transitions $c s_{x}$. Since only one of these case transitions fire, place $p_{\ominus}$ has only one token. $\square$

Lemma 4: Let $R$ be a rule set, and $N_{M}$ be the Petri net obtained by applying Algorithm 1-3 to $R$. The merged Petri net $N_{M}$ is $L_{1}$-live and deadlock-free.

Proof. To prove that the merged Petri net $N_{M}$ is 1-live, we show that for every transition in the Petri net, there is a sequence of markings that can fire it. Furthermore, we show that the $N_{m}$ is deadlock-free (i.e. there will be no remaining tokens such that no transition is enabled).

Since every task in the rule sets is contained in at least one derivation, based on Lemma 1, we have a sequence of markings that enables it and hence, based on Lemma 2, that task transition can fire in the merged $N_{M}$. We prove such a claim for different silent transitions that we have in $N_{M}$.

1) Start transition $t_{-} s$ can fire because the marking $m=\left\{p_{s}\right\}$ enables it (Line 8, Alg. 2 ).

2) Case transitions $c s_{x}$ can also fire because we have arcs $t_{-} s \mapsto x \mapsto c s_{x}$ (Line 3, Alg. 3). Thus, the marking sequence $m_{0}=\left\{p_{s}\right\}, m_{1}=\{x\}$ can fire transition $c s_{x}$.

3) Transition $t \_r$ corresponds to the rule $r: l_{1}, \ldots, l_{n} \Rightarrow l_{n+1}$. If this is the only rule with the head $l_{n+1}$, we know that we 
have a derivation $L_{1}, \ldots, L_{k}$ where $l_{1}, \ldots, l_{n}$ are in $L_{1}, \ldots, L_{k}$. By Lemma 2, we thus have a sequence of markings that can fire $t_{-} l_{1}, \ldots, t_{-} l_{n}$. After these transitions fire, since these are the only inputs of transition $t_{-} r$ with arcs $t_{-} l_{i} \mapsto p \mapsto t_{-} r$, transition $t_{-} r$ fires (Line 14-15, Alg. 2). Notice that if any of these transitions has different outputs in different Petri nets, we may have arcs $t_{-} l_{i} \mapsto x$ or $\mapsto C^{s} \mapsto p \mapsto t_{-} r$ (Line 30-34, Alg. 3). Transition $C^{s}$ has another input from corresponding case transition $c s_{x}$ and since this transition can fire, we can have a sequence of markings that fires $t \_r$ (Line 42-44, Alg. 3).

4) To handle the situation where we have more than one rule with the same head, say $r_{1}, \ldots r_{m}, r_{i}: l_{1}^{i}, \ldots, l_{n}^{i} \Rightarrow l_{n+1}$, we have transitions $t_{-} r^{\prime}$ and $t \_r^{\prime \prime}$ in the Petri net (Line 18-30, Alg. 2). In this case, we have a derivation $L_{1}, \ldots, L_{k}$ where $l_{1}^{i}, \ldots, l_{n}^{i} \mathrm{~s}$ are in $L_{1}, \ldots, L_{k}$. Therefore, by Lemma 2, we have a sequence of markings that can fire all $t_{-} l_{j}^{i} \mathrm{~s}$. After all $t_{-} l_{j}^{i} \mathrm{~s}$ have fired, all transitions $t_{-} r_{i}$ can fire and lead to a marking $m$. In this marking, we have $x_{i} \in m$. Accordingly, marking $m^{\prime}=m \cup\left\{p_{\oplus}\right\}$ can fire transition $t_{-} r_{i}^{\prime}$ (but only one). Furthermore, marking $m^{\prime \prime}=m-\left\{p_{\oplus}, x_{i}\right\}+\left\{p_{\sim}\right\}$ can fire transition $t_{-} r_{i}^{\prime \prime}$. Notice that it is also possible for transitions $t_{-} l_{j}^{i}$ to have different outputs in different Petri nets. The argument is the same as in (3).

5) $C^{s}$ transitions are added after task transitions during the merge when the outputs of those transitions are different in the Petri nets. As mentioned, we have a derivation for each task $t$, hence, by Lemma 2, we have a sequence of markings that fires $t$. If the outputs of $t$ are different in different Petri nets, we have arcs $t \mapsto x$ or $\mapsto C^{s}$ (Line 30-32, Alg. 3). Transition $C^{s}$ have another input from corresponding case transition $c s_{x}$ with arcs $c s_{x} \mapsto p^{\prime} \mapsto C^{s}$ (Line 42-44, Alg. 3). After transition $t$ fires, we have a token at place $x$ or and since the case transition $c s_{x}$ corresponding to the derivation can fire, we have a token at place $p^{\prime}$ too. Therefore, the corresponding $C^{s}$ can fire.

6) $C^{j}$ transitions are also added before task transitions during merge when the inputs of those transitions are different in the Petri nets (Line 12-19, Alg. 3). Given that we have a derivation for every task $t$, we have a marking sequence that fires transition $t$. As we argued in the proof of Lemma 2, the token in the input of this task comes from the $C^{j}$ transition corresponding to the case that derivation belongs to. Therefore, this sequence of markings fires that $C^{j}$ as well. Since every $C^{j}$ transition is for a derivation in different rule set, there is a marking sequence for each of them. However, considering inputs of $t_{-} e$ in different Petri nets, the inputs of it in one Petri net may be a subset of the inputs of it in other Petri nets. (Notice that this situation only occurs for $t \_e$ because all other task transitions have only one input and the inputs of other silent transition are always the same in all Petri nets). In this case, we may have $C^{j}$ before $t \_e$ with just one input from the corresponding case transition (Line 20-23, Alg. 3). $C^{j}$ can fire because the corresponding case transition can fire. 7) The other silent transitions that we have are transitions named $t_{C^{X}}$ (Line 35-41, Alg. 3). Whenever the outputs of a transition in one Petri net $N$ is subset of its outputs in other Petri nets, we have $C^{s}$ transitions with no output. These transitions are the inputs of $t_{C^{x}}$ transitions. Since we have a derivation including all tasks in the rule sets corresponding to Petri net $N$, all task transitions fire and thus all the $C^{s}$ transitions fire. Consequently, the corresponding $t_{C^{X}}$ can fire.
8) The inputs of $t \_e$ in each Petri net are from places $p_{\text {. }}$ for each set of rules with common head and also from task transitions $t$ that do not occur in the body of any rule (Line 3134, Alg. 2). Since we have a derivation $L_{1}, \ldots, L_{k}$ in the rule set which includes all tasks $t$, there is a marking sequence that fires all tasks and also as we argued for transitions $t \_r^{\prime \prime}$, it fires one of them. The place $p_{\mathbf{m}}$ is an xor place with inputs from all $t \_r^{\prime \prime}$ transitions (Line 29-30, Alg. 2); accordingly, it has a token and so do all the places after tasks which do not have any following transition to consume them (those which are not in the body of any rule). As a result $t_{-} e$ can fire in that Petri net. Since we have a trace in each Petri net that fires $t \_e$, we have a trace that fires $t \_e$ in $N_{M}$. Note that in $N_{M}, t \_e$ may have an input from $p_{\ominus}$. This place is an xor place with inputs from transitions $t_{C^{x}}$ or directly from case transitions (Line 45-51, Alg. 3). Based on the case the trace belongs to, the corresponding $t_{C^{X}}$ (if it exists) fires and puts a token in place $p_{\ominus}$. Otherwise, if $t_{C^{X}}$ does not exist, the token comes directly from the corresponding case transition. Therefore $t_{-} e$ can fire.

We now prove that there are no remaining tokens. Tokens remain in the inputs of transitions with more that one input. The only transitions with this condition are:

1) Transitions for rules. After $t_{-} e$ has fired, if we have a rule transition $t \_r$ with one or more remaining tokens, then the rule has not fired and, as a consequence, has not been used in the derivation corresponding to the current case $x$ firing $t \_e$. This remaining token comes from a task transition $t$ that has fired and this means that $t$ was in the derivation. Since $t$ is in the Petri net for case $x$, it has an output either to one of the rules other than $r$ or an output to $t_{-} e$ in that Petri net. Therefore, the outputs of $t$ are different in this Petri net compared to the Petri net in which it has an output to rule $r$. Accordingly, there should be an xor place after $t$ with arcs $t \mapsto$ xor $\mapsto C^{s}$ $\mapsto p \mapsto t \_r$ (Line 30-34, Alg. 3). Since the transition $C^{s}$ has an input from a case transition other than $x$, it cannot fire and thus the input place of $r$ from $t$ can never have a token.

2) Transitions $t_{-} r^{\prime}$ and $t_{-} r^{\prime \prime}$ for handling a set of rules with common head (Line 18-30, Alg. 2). Whenever we have a set of rules with common head in one case, in all derivations that fire $t \_e$, all these rules fire and consequently they all fire in Petri net $N_{M}$ as well. We thus have tokens in all places $x_{i} \mathrm{~s}$ and also we have a token in place $p_{\oplus}$. Now, one of transitions $t_{-} r_{i}^{\prime}$ can fire and consume the token in $p_{\oplus}$ and a token from corresponding $x_{i}$. The tokens in other $x_{i} \mathrm{~s}$ are consumed by a transition $t_{-} r^{\prime \prime}$. The inputs of that $t_{-} r^{\prime \prime}$ transition is from all $x_{i}$ s except the one for which its corresponding $t_{-} r^{\prime}$ has fired together with an input from $p_{\sim}$. Because the transition in the head of these rules fire and $p_{\sim}$ has a token, $t_{-} r^{\prime \prime}$ fires. Therefore, no tokens remain in inputs of $t_{-} r^{\prime}$ and $t_{-} r^{\prime \prime}$ transitions.

3) $t_{C^{x}}$ transitions (Line 35-41, Alg. 3 3). The inputs of $t_{C^{x}}$ are from $C^{s}$ transition with no output in case $x$. These transitions have an input from case transition $c s_{x}$; thus they all fire. Therefore, transition $t_{C^{X}}$ fires and consumes all the tokens in its input.

4) Transition $t \_e$ fires in all Petri nets and does so in $N_{M}$. Since the $N_{M}$ is 1-bounded (Lemma 3), whenever $t_{-} e$ fires, it consumes all input tokens and no tokens remain. 


\section{REFERENCES}

[1] N. Ghanbari Ghooshchi, N. R. T. P. van Beest, G. Governatori, F. Olivieri, and A. Sattar, "Visualisation of compliant declarative business processes," in Proceedings of EDOC, IEEE, 2017.

[2] T. Morgan, Business rules and information systems: aligning IT with business goals. Addison-Wesley Professional, 2002.

[3] G. Governatori and S. Sadiq, "The journey to business process compliance," in Handbook of Research on BPM (J. Cardoso and W. van der Aalst, eds.), ch. 20, pp. 426-454, IGI Global, 2009.

[4] W. M. P. Van Der Aalst, L. Aldred, M. Dumas, and A. H. M. ter Hofstede, "Design and implementation of the yawl system," in CAiSE, vol. 3084, pp. 142-159, Springer, 2004.

[5] N. R. T. P. van Beest, E. Kaldeli, P. Bulanov, J. C. Wortmann, and A. Lazovik, "Automated runtime repair of business processes," Information Systems, vol. 39, pp. 45-79, 2014.

[6] A. Marrella and M. Mecella, "Continuous planning for solving business process adaptivity," in 12th International Working Conference on Business Process Modeling, Development and Support (BPMDS-11), 2011.

[7] B. Heinrich and D. Schön, "Automated planning of process models: The construction of simple merges," in 24th European Conference on Information Systems (ECIS), 2016.

[8] J. Prescher, C. Di Ciccio, and J. Mendling, "From declarative processes to imperative models.," SIMPDA, vol. 14, pp. 162-173, 2014

[9] W. M. Van der Aalst, "The application of petri nets to workflow management," Journal of circuits, systems, and computers, vol. 8, no. 01, pp. 21-66, 1998.

[10] M. Hashmi, G. Governatori, and M. T. Wynn, "Normative requirements for regulatory compliance: An abstract formal framework," Information Systems Frontiers, vol. 18, no. 3, pp. 429-455, 2016.

[11] G. Governatori, F. Olivieri, A. Rotolo, S. Scannapieco, and M. Cristani, "Picking up the best goal - an analytical study in defeasible logic," in Proc. RuleML 2013, pp. 99-113, Springer, 2013.

[12] G. Governatori, F. Olivieri, S. Scannapieco, A. Rotolo, and M. Cristani, "The rationale behind the concept of goal," TPLP, vol. 16, no. 3 , pp. 296-324, 2016.

[13] M. Ghallab, D. Nau, and P. Traverso, Automated Planning: Theory and Practice. Morgan Kaufmann, 2004.

[14] W. M. P. van der Aalst, "The application of petri nets to workflow management," Journal of Circuits, Systems, and Computers, vol. 8, no. 1, pp. 21-66, 1998.

[15] C. A. Petri, Communication with automata. $\mathrm{PhD}$ thesis, Universität Hamburg, 1966

[16] T. Murata, "Petri nets: Properties, analysis and applications," Proceedings of the IEEE, vol. 77, no. 4, pp. 541-580, 1989

[17] R. Dijkman, M. Dumas, and C. Ouyang, "Semantics and analysis of business process models in bpmn," Information and Software Technol$o g y$, vol. 50 , no. 12 , pp. 1281-1294, 2008

[18] S. J. J. Leemans, D. Fahland, and W. M. P. van der Aalst, "Discovering block-structured process models from event logs - a constructive approach," in International Conference on Applications and Theory of Petri Nets and Concurrency, pp. 311-329, Springer, 2013.

[19] H. M. W. Verbeek, J. Buijs, B. F. Van Dongen, and W. M. P. van der Aalst, "Prom 6: The process mining toolkit," BPM Demos, vol. 615 pp. 34-39, 2010.

[20] L. García-Bañuelos, N. R. T. P. van Beest, M. Dumas, M. La Rosa, and W. Mertens, "Complete and interpretable conformance checking of business processes," IEEE Transactions on Software Engineering, 2017.

[21] J. De Weerdt, J. Vanthienen, B. Baesens, et al., "An improved process event log artificial negative event generator," Tech. Rep, 2012

[22] H. Groefsema, N. R. T. P. van Beest, and M. Aiello, "A formal model for compliance verification of service compositions," IEEE Transactions on Services Computing, 2016.

[23] M. Dastani, M. B. van Riemsdijk, and J. C. Meyer, "Programming multi-agent systems in 3APL," in Multi-Agent Programming: Languages, Platforms and Applications, pp. 39-67, 2005.

[24] M. Dastani, "2apl: a practical agent programming language," $A u-$ tonomous Agents and Multi-Agent Systems, vol. 16, no. 3, pp. 214-248, 2008.

[25] R. H. Bordini and J. F. Hübner, "BDI agent programming in agentspeak using Jason (tutorial paper)," in CLIMA VI, pp. 143-164, 2005.

[26] J. Broersen, M. Dastani, J. Hulstijn, and L. W. N. van der Torre, "Goal generation in the BOID architecture," Cognitive Science Quarterly, vol. 2, no. 3-4, pp. 428-447, 2002.

[27] N. Alechina, N. Bassiliades, M. Dastani, M. D. Vos, B. Logan, S. Mera, A. Morris-Martin, and F. Schapachnik, "Computational models for normative multi-agent systems," pp. 71-92.

[28] N. Alechina, M. Dastani, and B. Logan, "Programming norm-aware agents," pp. 1057-1064.
[29] A. C. Kakas, P. Mancarella, F. Sadri, K. Stathis, and F. Toni, "The KGP model of agency," in ECAI, pp. 33-37, IOS Press, 2004.

[30] A. Gabaldon, "Making golog norm compliant," in CLIMA (J. Leite, P. Torroni, T. Ågotnes, G. Boella, and L. van der Torre, eds.), vol. 6814 of Lecture Notes in Computer Science, pp. 275-292, Springer, 2011.

[31] M. Pesic, H. Schonenberg, and W. M. Van der Aalst, "Declare: Full support for loosely-structured processes," in Enterprise Distributed Object Computing Conference, 2007. EDOC 2007. 11th IEEE International, pp. 287-287, IEEE, 2007.

[32] M. Westergaard and F. M. Maggi, "Declare: A tool suite for declarative workflow modeling and enactment.," BPM (Demos), vol. 820, pp. 1-5, 2011.

[33] G. De Giacomo, M. Dumas, F. M. Maggi, and M. Montali, "Declarative process modeling in BPMN," in International Conference on Advanced Information Systems Engineering, pp. 84-100, Springer, 2015.

[34] G. Governatori and M. Hashmi, "No time for compliance," in Proc. of the Int. Conf. on Enterprise Distibuted Object Computing, pp. 9-18, IEEE, 2015.

[35] S. Sardiña and L. Padgham, "A BDI agent programming language with failure handling, declarative goals, and planning," Autonomous Agents and Multi-Agent Systems, vol. 23, no. 1, pp. 18-70, 2011.

[36] F. Olivieri, G. Governatori, S. Scannapieco, and M. Cristani, "Compliant business process design by declarative specifications," in Proc. PRIMA 2013, pp. 213-228, 2013.

[37] F. Olivieri, M. Cristani, and G. Governatori, "Compliant business processes with exclusive choices from agent specification," in Proc. PRIMA 2015, pp. 603-612, 2015.

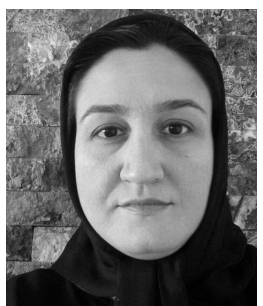

Nina Ghanbari Ghooshchi is a Ph.D. candidate at Griffith University, Nathan, Australia. She received her M.Sc. degree in Artificial Intelligence in 2006 from Sharif University of Technology, Iran. Her research interests include artificial intelligence, automated planning and business process compliance. She currently collaborates with the Computational Law and Legal Informatics team at Data61, CSIRO working on automated generation of business processes from business rules.

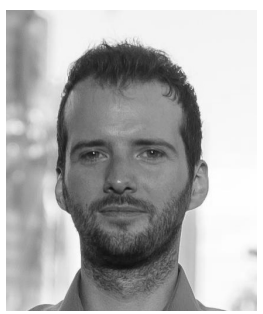

Nick van Beest is a Research Scientist at Data61, CSIRO in Brisbane, Australia. He obtained his PhD in Information Systems in 2013 at the University of Groningen, The Netherlands. His research experience covers artificial intelligence, business process compliance and process mining. He currently works on automated generation of business processes from laws and business rules, in order to ensure continuous compliance. In addition, he is working on declarative variability management.

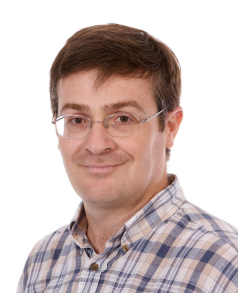

Guido Governatori leads the Software Systems group and the research activities on Computational Law and Legal Informatics at Data61, CSIRO. He received his $\mathrm{PhD}$ in Legal Informatics from the University of Bologna. His research areas include nonclassical logics and their applications to legal reasoning, autonomous agents, and business processes. He won the 2016 Gold Award for the Australian Computer Society ICT Researcher of the Year.

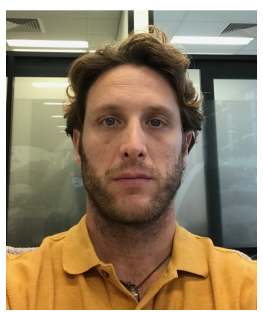

Francesco Olivieri is a Postdoctoral Research Fellow at Data61, CSIRO in Brisbane, Australia. Francesco obtained a double $\mathrm{PhD}$ with the Griffith University, Australia, and The University of Verona, Italy. His research areas are in Artificial Intelligence, knowledge representation, non-monotonic reasoning, business process compliance, and deontic logics. He is currently working on a new logical formalism to capture notions of loops and resource consumption within business process models. 